\title{
Spatio-Temporal Analysis of Foraging Behaviors of Anelosimus studiosus Utilizing Mathematical Modeling of Multiple Spider Interaction on a Cooperative Web
}

\author{
Alex John Quijano ${ }^{\mathrm{a}}$, Michele L. Joyner ${ }^{\mathrm{a}, \mathrm{b}}$, Chelsea Ross ${ }^{\mathrm{a}, \mathrm{c}}$, Colton Watts ${ }^{\mathrm{c}}$, \\ Edith Seier ${ }^{\mathrm{a}, \mathrm{b}}$, Thomas C. Jones ${ }^{\mathrm{c}}$ \\ ${ }^{a}$ Department of Mathematics \& Statistics \\ PO Box 70663 \\ East Tennessee State University, Johnson City, TN, USA 37614 \\ ${ }^{b}$ Computation and Research in Data Science (CaRDS) Institute \\ East Tennessee State University, Johnson City, TN, USA \\ ${ }^{c}$ Department of Biological Sciences \\ East Tennessee State University, Johnson City, TN, USA
}

\begin{abstract}
In this paper, we develop a model for predation movements of a subsocial spider species, Anelosimus studiosus. We expand on a previous model to include multiple spider interaction on the web as well as a latency period during predation. We then use the model to test different spatial configurations to determine the optimal spacing of spiders within a colony for successful capture during predation. The model simulations indicate that spiders uniformly spacing out along the edge of the web results in the most successful predation strategy. This is similar to the behavior observed by Ross [25] in which it was determined to be statistically significant that during certain times of the day, spiders were positioned along the edge more than expected under complete spatial randomness.
\end{abstract}

Keywords: Mathematical Modeling, Stochastic Differential Equation, Multiple Species Interaction, Anelosimus studiosus, Foraging Behavior

Email addresses: aquijano4@ucmerced.edu (Alex John Quijano), joynerm@etsu.edu (Michele L. Joyner), seier@etsu.edu (Edith Seier), jonestc@etsu.edu (Thomas C. Jones)

Preprint submitted to Journal of Theoretical Biology

August 9, 2016

(C) 2016. This manuscript version is made available under the Elsevier user license http://www.elsevier.com/open-access/userlicense/1.0/ 


\section{Introduction}

Hunting cooperatively can benefit predators both by increasing search efficiency (local enhancement) and in the collective ability to subdue prey [6]. Improving search efficiency is particularly important when resources are scarce and patchily distributed, but only promotes cooperative hunting when resources, once found, are sufficient to feed the group [28, 29]. Some examples include subterranean mole rats searching for large but scarce tubers [10], and vultures searching for carcasses on the savanah [27]. Once prey is located, cooperative hunting allows groups to more effectively chase down and subdue larger prey [17], well known in social animals such as lions [26] and wolves [15]. The examples above are from neurologically complex vertebrate animals which have evolved sophisticated communication and social organization allowing for coordination among group members in searching and subduing prey. In this study we explore how neurologically simpler animals without well-developed communication and coordination mechanisms might also take advantage of cooperative hunting.

Sociality in spiders occurs in only a few dozen of over 45,000 spider species [8], but it has evolved multiple times in mostly tropical habitats, including multiple times within the genus Anelosimus [1]. Social spiders build communal webs in which they hunt cooperatively and communally care for broods [14]. Similar to cooperatively hunting mammals, social spiders are able to attack in groups, subduing larger prey for their size than can solitary spiders [30]. Social spiders also maintain larger webs potentially intercepting more prey [23]. In this study we specifically test how different spatial distributions of spiders in a communal web affect the likelihood of the spiders being able to subdue intercepted prey before they escape.

A. studiosus is a spider that varies in its level of sociality among populations across its range. Most populations are subsocial with colonies consisting of a single mother and her offspring' however toward the northern edge of their range, there are social colonies of multiple adult females cooperating in prey capture, web maintenance, and brood care [11]. These spiders are cobweb weavers in which the web consist of knockdown threads, a capture sheet and a retreat. The knockdown threads cause the flight path of the prey to be disrupted, causing them to fall onto the capture sheet. Unlike orb weavers, the capture sheet is not sticky; therefore, if the prey can reach the edge of 
the web, they can escape. In a paper by Ross [25] (which is summarized in Section 7), experimental data indicated that spiders within these types of colonies changed their positioning throughout the day; they were clustered at certain times while they were spread out and closer to the edges of the web at other times of the day. The hypothesis, first investigated by Joyner et. al. [2], was that spiders must stay close to the retreat when the threat of predators is high; however, they spread out to optimize prey capture during other parts of the day. The model in [2] was a more simplistic model for predation in which it was assumed that spiders acted immediately upon detection of a prey in the web and moved in a similar manner to each other with no interaction between the spiders of the web. Under these assumptions, they concluded that the "model predictions generally support the hypothesis that these spiders can improve their communal prey capture success employing regular or random distributions, thus increasing their collective foraging area. The model also predicted that if the spiders position themselves around the perimeter of the web they are more successful at capturing prey" [2].

The original model was modified in the paper by Quijano et. al. [22] to more accurately include the differences seen in spiders during prey capture. They developed an aggregate model which incorporated variation in spider movement. With the modified model, one can now simulate the differences seen among spiders experimentally. However, since A. studiosus is a subsocial spider species, the spiders work together in foraging and web maintenance. In this case, we hypothesize that the movements of a single spider should be influenced by the other spiders in the web as well as the prey. Moreover, experimental data indicates that not all spiders immediately act when a prey lands in the web; a latency period is observed. This latency period could affect the success of prey capture. This paper focuses on the development and implementation of an extended aggregate stochastic model which takes into account the interaction of multiple spiders during prey capture and the incorporation of a latency period. We then use the model under different initial spatial configurations and under different levels of influence from the other spiders in the web to determine their effect on the probability of successful foraging. In Sections 2 and 3, we summarize the mathematical model of a single spider and prey during predation. In Section 4, we develop the methods used to incorporate the influence of other spiders in the web during prey capture. Section 5 outlines the various spatial configurations used for simulation purposes. The results of the simulation studies for each of the configurations for a differing number of spider colonies is given in Section 
6. In Section 7, we summarize the experimental results from [25] describing the spatial positioning of Anelosimus studiosus across the day and age of development. We given some concluding remarks in Section 8.

\section{Mathematical Model of Single Foraging Spider}

The predation behaviors of the subsocial spider Anelosimus studiosus has been modeled by Joyner et. al. [2] and Quijano et. al. [22] using stochastic differential equations and computational algorithms. In [2], the trajectory of the spider is determined by

$$
d \mathbf{r}(t)=\mu\{\mathbf{r}(t), t\} d t+\Sigma\{\mathbf{r}(t), t\} d \mathbf{W}(t)
$$

where $\mathbf{r}(t)=\left[X_{s}(t), Y_{s}(t)\right]^{\prime}$ represents the location of the spider at time $t$, $d t$ is an incremental change in time, $\mu$ is the directional component, $\Sigma$ is a diffusion parameter, and $\mathbf{W}$ is assumed to be a brownian process [2]. The diffusion parameter is given by

$$
\Sigma\{\mathbf{r}(t), t\}=\left[\begin{array}{cc}
\sigma_{x} & 0 \\
0 & \sigma_{y}
\end{array}\right]
$$

where it is assumed $\sigma_{x}=\sigma_{y}=\sigma$. Furthermore, the drift parameter is specified by

$$
\mu\{\mathbf{r}(t), t\}=v_{s}\left[\begin{array}{c}
\cos \left(\theta_{o}\left(t_{i}\right)+\epsilon\left(t, d_{i}\right)\right) \\
\sin \left(\theta_{o}\left(t_{i}\right)+\epsilon\left(t, d_{i}\right)\right)
\end{array}\right]
$$

where $v_{s}$ is the spider's velocity, $\theta_{o}\left(t_{i}\right)$ is the optimal direction, i.e., the direction the spider would go if the spider went directly to the prey, and $\epsilon\left(t, d_{i}\right)$ gives the error in direction. By substituting the above terms into Equation (1), the stochastic differential equation is given by

$$
\left[\begin{array}{l}
d X_{s}(t) \\
d Y_{s}(t)
\end{array}\right]=v_{s}\left[\begin{array}{c}
\cos \left(\theta_{o}\left(t_{i}\right)+\epsilon\left(t, d_{i}\right)\right) \\
\sin \left(\theta_{o}\left(t_{i}\right)+\epsilon\left(t, d_{i}\right)\right)
\end{array}\right] d t+\left[\begin{array}{cc}
\sigma & 0 \\
0 & \sigma
\end{array}\right] d \mathbf{W}(t) .
$$

The error in direction $\epsilon$ is drawn from a normal distribution [22] with mean

$$
e(d)=\beta_{3} d^{3}+\beta_{2} d^{2}+\beta_{1} d+\beta_{0}
$$

and standard deviation $q_{1}$ for distances $8 \mathrm{~cm} \leq d \leq 11 \mathrm{~cm}$ and $q_{2}$ for $d<8$ $\mathrm{cm}$. We note that in the formula for $e(d), \beta_{0}, \beta_{1}, \beta_{2}$, and $\beta_{3}$ are constants estimated in [22] using experimental data and $d$ is the distance between the 
spider and prey. At distances greater than $12 \mathrm{~cm}$, the error function is set equal to one radian to prevent the error from increasing indefinitely for large distances which could cause the simulated spider to never capture the prey.

Pauses and varying velocities are included in the model using an algorithm developed by Quijano et. al. [22] shown in Figure 1. The algorithm has two main loops, the pause loop (when $v_{s}=0$ ) and the run loop (when $v_{s}>0$ ). In the algorithm, $D_{p}$ and $D_{r}$ denote the durations of the pause and run respectively, and $T_{p}$ and $T_{r}$ are the timers for the pause and run. We assume the duration of the pause is drawn from an exponential distribution with mean $q_{3}$, the velocity varies according to a uniform distribution with mean $q_{4}$, and the duration of the run follows a truncated normal distribution with mean $q_{5}$ and degree of variance $q_{6}$. Justifications for the chosen distributions can be found in [22] where a linear relationship was also established between $q_{1}$ and $q_{2}$,

$$
q_{2}=\alpha_{0}+\alpha_{1} q_{1},
$$

and an exponential relationship was established between $q_{5}$ and $q_{6}$,

$$
q_{6}=\gamma e^{\lambda q_{5}} .
$$

A summary of parameter definitions can be found in Table 1.

Table 1: Summary of parameters.

\begin{tabular}{cc}
\hline Definition & Symbol \\
\hline Mean error for long distances & $q_{1}$ \\
\hline Mean error for short distances & $q_{2}$ \\
\hline Mean duration of pause & $q_{3}$ \\
\hline Mean velocity & $q_{4}$ \\
\hline Mean duration of run & $q_{5}$ \\
\hline Degree of variance & $q_{6}$ \\
\hline
\end{tabular}

Since a subsocial spider colony is typically composed of a mother and her juveniles, it is necessary to consider the juvenile as well. We make a few minor adjustments to the model in the case of a juvenile spider. Our first assumption is that juveniles cannot run as fast as the adult mother; therefore, if the spider is a juvenile, the mean velocity of the juvenile is reduced to a quarter of the mother's mean velocity, i.e. $\frac{1}{4} q_{4}$. Furthermore, 


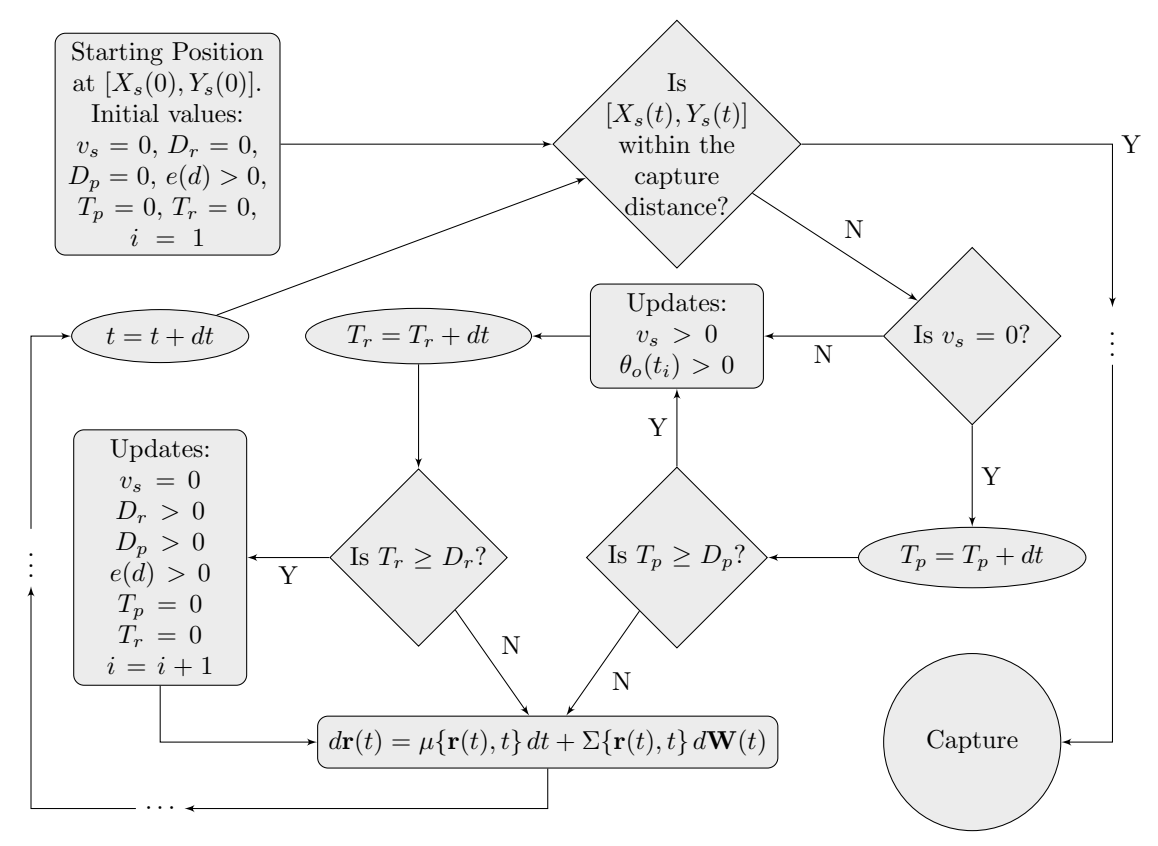

Figure 1: The Stochastic Model Algorithm.

for an adult spider, it is assumed that if a spider is within a certain capture distance from the prey, it is not possible for the prey to escape and is therefore considered captured. We extend the capture criteria for multiple spiders depending on who falls within the capture distance. If the mother is within the capture distance, then the prey is considered captured regardless of how many juvenile are within the capture distance. If the mother is not within the capture distance, there must be at least three juveniles within the capture distance in order for the prey to be considered captured. Basically, it takes three or more juvenile spiders to take down a prey while an adult spider can take down a prey by itself [2]. In the next section, we consider the movement of the prey.

\section{Mathematical Model of Prey}

It is important to model the prey since it is the main trajectory point which the spiders need to locate. The movement of the prey has never been studied closely when being hunted by A. studiosus. Theoretically, the prey moves in different directions depending upon whether they are aware they are being hunted or not. Therefore, we consider two types of prey for the purpose 
of simulating the predation movements of $A$. studiosus, namely perceptive and careless prey as done in [2]. Assuming, the movement of the prey is modeled similar to the spider, the stochastic differential equation for the prey movement is given by

$$
\left[\begin{array}{l}
d X_{p}(t) \\
d Y_{p}(t)
\end{array}\right]=v_{p}\left[\begin{array}{c}
\cos \left(\theta_{p}\left(t_{i}\right)\right. \\
\sin \left(\theta_{p}\left(t_{i}\right)\right.
\end{array}\right] d t+\left[\begin{array}{ll}
\sigma & 0 \\
0 & \sigma
\end{array}\right] d \mathbf{W}(t)
$$

where $v_{p}$ is the velocity of the prey, $\theta_{p}\left(t_{i}\right)$ is the angle of incidence at an update time $t_{i}$, and $\mathbf{W}$ is the assumed brownian process. The velocity of the prey is given by a fixed value, $v_{p}=0.2557 \mathrm{~cm} / \mathrm{s}$, and the diffusion component is given by $\sigma=0.25[2]$. The duration of time until the prey changes its direction is taken from an exponential distribution with mean of 5 seconds [2].

The perceptive prey is a type of prey that is aware that it is being hunted as soon as it lands on the web. It locates the nearest edge and walks accordingly to escape. Let $\mathrm{P}(\mathrm{t})=\left[X_{p}(t) Y_{p}(t)\right]$ indicate the $x$ and $y$ coordinates of the prey at time $t$. Assume the web is square with length $\diamond$ and the bottom left corner is located at the point $(0,0)$. In assuming the prey walks to the closest edge, the prey will immediately walk either left to the point $\left(0, Y_{p}(t)\right)$, straight to the point $\left(X_{p}(t), \diamond\right)$, right to the point $\left(\diamond, Y_{p}(t)\right)$ or back to the point $\left(X_{p}(t), 0\right)$ (see Figure 2). Let $E(t)$ be a matrix whose rows $E_{i}(t)$ consist of the location of these adjacent points on the edge at time $t$, i.e.,

$$
E(t)=\left[\begin{array}{cc}
0 & Y_{p}(t) \\
X_{p}(t) & \diamond \\
\diamond & Y_{p}(t) \\
X_{p}(t) & 0
\end{array}\right] .
$$

We want to calculate the distances from the prey to the four edges and take the minimum distance. Let $l$ be a vector of distances from the adjacent edge points to the prey at time $t$ with components given by

$$
l_{i}(t)=\left\|E_{i}(t)-P(t)\right\|
$$

for rows $(i)=\{1,2,3,4\}$. Let $e_{m}(t)$ be the row index such that $\min (l(t))=$ $l_{e_{m}}(t)$. Thus, $E_{e_{m}}(t)$ indicates the point towards which the prey will travel. We assume the mean direction is towards this point with error in the direction of variance 1 . Therefore the angle of incidence of a perceptive prey at time 
$1 \quad t$ is given by

$$
\theta_{p}\left(t_{i}\right) \sim \tan ^{-1}\left(\frac{N\left(E_{e_{m}}(t), 1\right)-P(t)}{\left\|N\left(E_{e_{m}}(t), 1\right)-P(t)\right\|}\right)
$$

3 where $N(a, b)$ represents the normal distribution with mean $a$ and variance $4 b$.

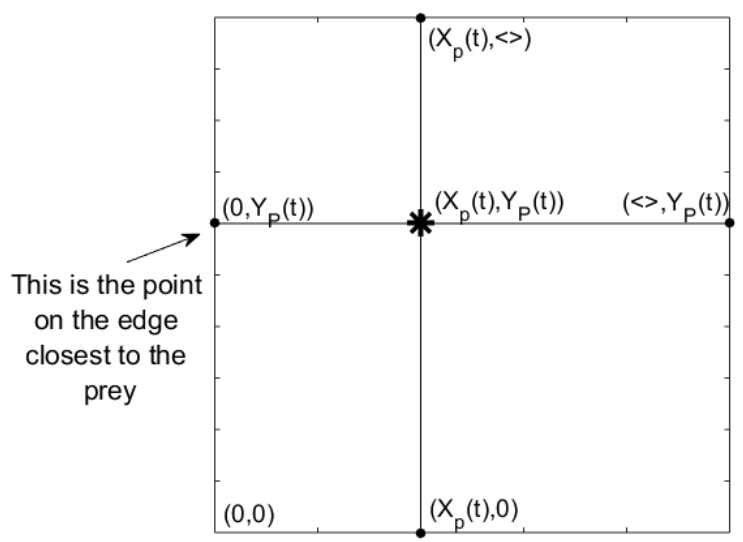

Figure 2: Perceptive Prey

The careless prey is a type of prey that is unaware that it is being hunted. Assuming the careless prey can move everywhere with all of the direction are equally likely, the angle of incidence of a careless prey is given by

$$
\theta_{p}\left(t_{i}\right) \sim U(0,2 \pi)
$$

where $U(0,2 \pi)$ is a uniform distribution with upper and lower bounds of 0 and $2 \pi$.

\section{Mathematical Modeling of Multiple Spiders' Interaction}

Since a colony of subsocial spiders share the same web, the influence of the surrounding objects could affect the direction of the spiders. In order to model the interaction of the spiders on the web, we incorporate aspects of the model developed in the paper by Heppner et. al. [9] which investigated the movement of coordinated bird flocks flying towards their home nest. Using similar terminology for the behavior of A. studiosus during predation as that used for the coordinated bird flocks [9], for multiple spiders in the web, we add two important aspects to the model: 
- Homing: The spiders will travel towards the perceived location of the prey (recall there is error in the spider's perceived location of the prey and the prey's actual location). The perceived location of the prey will be determined based on both the influence from the vibrations thought to be that of the prey as well as the directional cues given from other spiders in the web.

- Interaction: For a particular spider, the level of influence from the other spiders in the web is based on the distances to the other spiders.

Recall that the directional component of Equation (2) has an angle of incidence for spider $u \epsilon\{1,2, \ldots, n\}$ is given by

$$
\iota_{u}=\theta_{o}\left(t_{i}\right)_{u}+\epsilon\left(t_{i}, d\right)_{u}
$$

where $\iota_{u}$ is the angle of incidence influenced by the prey, $\theta_{o}\left(t_{i}\right)_{u}$ is the optimal angle to the prey, and $\epsilon\left(t_{i}, d\right)_{u}$ is the error function (Eq. (3)). This angle of incidence directs the spider to its perceived location of the prey. This is where they sense the vibration from the prey. The spider does not know exactly where the prey is initially, but the angle of incidence is where they need to look for the prey. This is under the assumption that the prey has a full level of influence without any influence from other spiders.

\subsection{Perceived Location of Prey}

Consider multiple spiders on the web and the spiders are moving. Since the spiders detects the prey through vibrations, the success of finding the prey depends on how much the prey produce vibrations along with the other spiders vibrations. We assume that the angle of incidence is dependent on the prey's level of influence. We define the prey's level of influence to be $0 \leq \omega_{p} \leq 1$ where 0 is no influence and 1 is where there is full influence from the prey. Note that if $\omega_{p}=1$, then the spider will know exactly where to search for the prey. If $\omega_{p}<1$, then the range of search increases since the level of influence is weaker. Suppose $\omega_{p}<1$, then the spider has to search for the prey at a range depending upon the prey's level of influence. This is called the range of vibration localization. This is the range of where a spider should look for the prey after they felt a vibration in an area. The range of the angle of incidence is given by

$$
\iota_{u} \pm \pi\left(1-\omega_{p}\right) .
$$




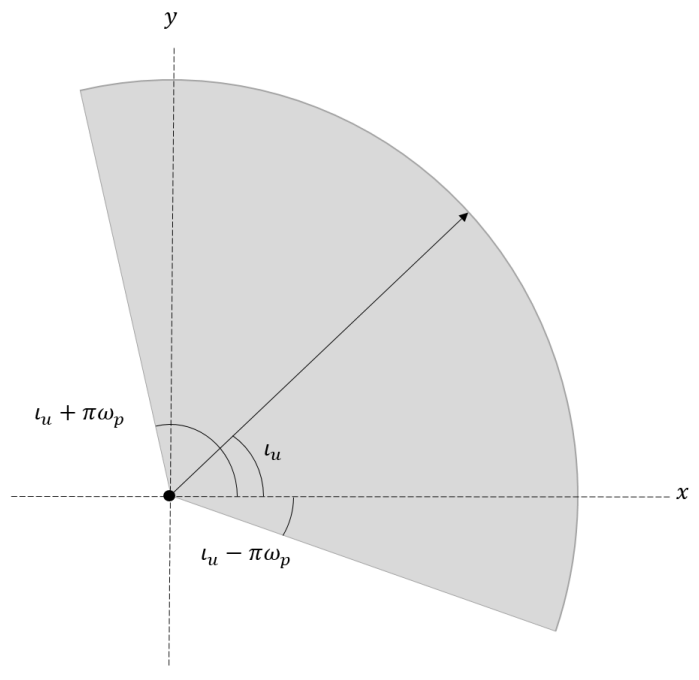

Figure 3: Range of localization.

(1)

(1)

Figure 3 shows an illustration of the range of localization where $\iota_{u}$ is the angle of incidence and the interval $\left(\iota_{u}-\left(1-\omega_{p}\right), \iota_{u}+\left(1-\omega_{p}\right)\right)$ is the range. The spider will search for the prey in all directions within the range of localization. Assuming all directions within the range are equally likely and $\omega_{p}<1$, the angle of incidence for spider $u \in\{1,2, \ldots, n\}$ is taken from a uniform distribution from $\iota_{u}-\left(1-\omega_{p}\right)$ to $\iota_{u}+\left(1-\omega_{p}\right)$ which is given by

$$
\phi_{u} \sim U\left(\iota_{u}-\left(1-\omega_{p}\right), \iota_{u}+\left(1-\omega_{p}\right)\right) .
$$

If $\omega_{p}=1$, then the range is $\left(\iota_{u}-0, \iota_{u}+0\right)$. If $\omega_{p}=0$, then the range is $\left(\iota_{u}-\pi, \iota_{u}+\pi\right)$ which is the full unit circle from 0 to $2 \pi$. Let $S$ be an $n \times 2$ vector containing the location of spider $u \in\{1,2, \ldots, n\}$. The matrix $S$ is given by

$$
S=\left[\begin{array}{cc}
X_{1} & Y_{1} \\
X_{2} & Y_{2} \\
\vdots & \vdots \\
X_{n} & Y_{n}
\end{array}\right]
$$

Then the location perceived for the prey by spider $u$ is given by

$$
\rho_{u}=S_{u}+d_{u}\left[\sin \left(\phi_{u}\right) \cos \left(\phi_{u}\right)\right]
$$


where $d_{u}$ is the distance from spider $u$ to prey and $S_{u}=\left[X_{u}(t) Y_{u}(t)\right]_{u}$ is the current location of spider $u$ at time $t$.

Now we consider the effect of other spiders on the web as well. Each object on the web contributes to the final direction of a spider. Assuming the prey and the spiders are the only objects on the web, each object has a level of influence based upon the amount of movement detected. We assume the spiders know, at some level, the difference between the movement of the prey versus the movement of the other spiders on the web; therefore we consider these levels of influence differently. Above, we discussed the effect of the influence of the prey. For multiple spiders, we assume the level of influence of other spiders on the web is directly related to the distance between the spiders.

Let $n$ be the number of spiders on a web. Let $S_{u}=\left[X_{s} Y_{s}\right]_{u}$ be the position of the spider at time $t$ for spider $u \epsilon\{1,2, \ldots, n\}$. We leave out the term $t$ since it is implied in the equation. Let $\delta$ be an $n \times n$ distance matrix at time $(t)$ given by

$$
\delta=\left[\begin{array}{cccc}
\delta_{11} & \delta_{12} & \cdots & \delta_{1 n} \\
\delta_{21} & \delta_{22} & \cdots & \delta_{1 n} \\
\vdots & & \ddots & \vdots \\
\delta_{n 1} & \delta_{n 2} & \cdots & \delta_{n n}
\end{array}\right]
$$

where

$$
\delta_{u v}=\left\|S_{u}-S_{v}\right\|
$$

is the distances from spider $u \in\{1,2, \ldots, n\}$ to spider $v \in\{1,2, \ldots, n\}$. If $u=v$, then the diagonals should be equal to zero since a spider is zero distance to itself.

Now suppose there are 2 spiders $S_{1}$ and $S_{2}$ with a prey $P$, see Figure 4 ,. The angle of incidence $\phi_{1}$ and $\phi_{2}$ points towards the perceived locations, $\rho_{1}$ and $\rho_{2}$, of the prey. We define $\phi_{12}$ as the angle of incidence from spider $S_{1}$ to the perceived location of the prey according to spider $S_{2}$. Similarly, $\phi_{21}$ is the angle of incidence from spider $S_{2}$ to the perceived location of the prey according to spider $S_{1}$. Recall the perceived location of each spider is given by Eq. (12). Then the angle of incidence of a spider based on the perceived locations of the other spiders, is calculated by

$$
\phi_{u v}=\tan ^{-1}\left(\frac{\rho_{v}-S_{u}}{\left\|\rho_{v}-S_{u}\right\|}\right) .
$$


1 We let $\Phi$ be an $n \times n$ matrix containing the angles of incidence based on the 2 perceived locations of the other spiders,

$$
\Phi=\left[\begin{array}{cccc}
\phi_{11} & \phi_{12} & \cdots & \phi_{1 n} \\
\phi_{21} & \phi_{22} & \cdots & \phi_{2 n} \\
\vdots & \vdots & \ddots & \vdots \\
\phi_{n 1} & \phi_{n 2} & \cdots & \phi_{n n}
\end{array}\right]
$$

4 The diagonal of $\Phi$ is equal to $\phi_{u}$ given in Equation (11) since this is the angle 5 of incidence of spider $u$ to the prey. The other entries of $\phi$ indicate the angle 6 of incidence from spider $u$ to the perceived location of the prey according to 7 spider $v$.

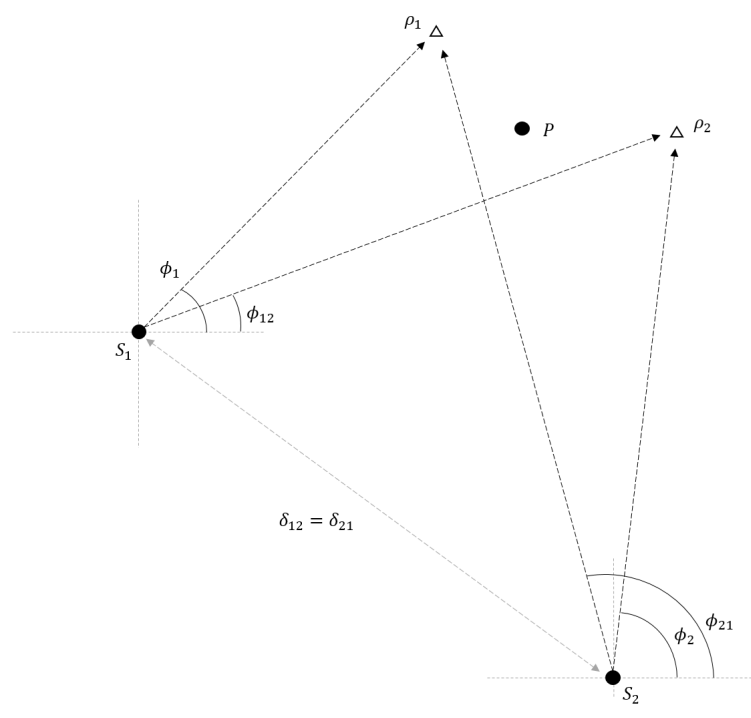

Figure 4: Location Perception Illustration.

\subsection{Latency Period}

Before we implement the extended model for multiple spiders into the SDE, we must include the latency period of each spider. The latency period is the amount of time the spider waits until it decides to pursue the prey after the prey lands on the web. Once a spider is moving, the other spiders will start to realize that it is time to move. Thus, the latency period is influenced by how many spiders are moving. The latency period of each 
1

spider is different. Using data given in [22], it was determined that the latency period across multiple spiders satisfies an exponential distribution with mean 16.81s. Initially, there are no moving spiders. The extended model for multiple spiders is used only if more than one spider is moving.

Let $M$ be a 'moving vector' of length $n$. The moving vector is a binary indicator for each spider indicating whether the spider is moving " 1 " or it is still at rest "0". The initial moving vector where all spiders are initially at rest is given by

$$
M_{o}=\left[\begin{array}{c}
0 \\
0 \\
\vdots \\
0
\end{array}\right] .
$$

The final moving vector where all of the spiders are moving is

$$
M_{f}=\left[\begin{array}{c}
1 \\
1 \\
\vdots \\
1
\end{array}\right] .
$$

We order the spiders so spider 1 is the first to start moving, spider 2 is the second, etc. We assume the first spider will wait the entire latency period before moving; however, we assume that after the first spider has started moving, the wait time for the second spider to initially move will be reduced by a factor of $\frac{1}{n}$ where $n$ is the number of spiders in the web. Therefore, if the latency period for spiders 1 and 2 (as initially drawn from the exponential distribution) are initially given by $\tau_{1}^{(0)}$ and $\tau_{2}^{(0)}$ respectively, then the latency period remaining before spider 2 moves is given by

$$
\tau_{2}^{(1)}=\left(1-\frac{1}{n}\right)\left(\tau_{2}^{(0)}-\tau_{1}^{(0)}\right)
$$

(the time remaining in second spider's latency period is reduced by $\frac{1}{n}$ ). Similarly, the latency period for all the remaining spiders $u \in\{2, \ldots, n\}$ is reduced according to

$$
\tau_{u}^{(1)}=\left(1-\frac{1}{n}\right)\left(\tau_{u}^{(0)}-\tau_{1}^{(0)}\right) .
$$

However, once spider 2 moves, we assume there is a further reduction in the remaining latency time for all the spiders (since more spiders are moving). 
The latency period is now reduced by a factor of $\frac{2}{n}$. Therefore, we have for $u \in\{3, \ldots, n\}$,

$$
\tau_{u}^{(2)}=\left(1-\frac{2}{n}\right)\left(\tau_{u}^{(1)}-\tau_{2}^{(1)}\right)
$$

where $\tau_{u}^{(1)}-\tau_{2}^{(1)}$ is the amount of the latency period already passed. In general, if $r$ spiders are moving, the updated latency period remaining for spiders $\{r+1, \ldots, n\}$ is given by

$$
\tau_{u}^{(r)}=\left(1-\frac{r}{n}\right)\left(\tau_{u}^{(r-1)}-\tau_{r}^{(r-1)}\right) .
$$

\subsection{Level of Influence}

Finally, we need to incorporate the level of influence each moving spider in the web has on the other moving spiders in the web. The level of influence is a measure of how much an object influences the movement and the direction of the spider. We again emphasize that when a spider moves (latency period) and the direction in which it moves (perceived location) are both influenced by other spiders in the web according to the level of influence neighbors have on its movement. Assuming the level of influence of the spiders depends on the distances between them, if two spiders are close to each other then those spiders would have more influence on each other's movement than the spiders located at longer distances. Let $\Omega$ be an $n \times n$ matrix at time $(t)$,

$$
\Omega=\left[\begin{array}{cccc}
\omega_{11} & \omega_{12} & \cdots & \omega_{1 n} \\
\omega_{21} & \omega_{22} & \cdots & \omega_{2 n} \\
\vdots & \vdots & \ddots & \vdots \\
\omega_{n 1} & \omega_{n 2} & \cdots & \omega_{n n}
\end{array}\right]
$$

where the entry $\omega_{u v}$ indicates the level of influence from spider $u \epsilon\{1,2, \ldots, n\}$ to spider $v \in\{1,2, \ldots, n\}$ and is given by

$22 \omega_{u v}=\left\{\begin{array}{cc}\frac{1-\omega_{p}}{\sum_{k=1, k \neq v}^{n}\left(\frac{\delta_{u v}}{\delta_{k v}}\right)^{2}} & u \neq v \\ \omega_{p} & u=v\end{array}\right.$

where $\omega_{p}$ indicates the level of influence of the prey. We note that if $u=v$, the only influence is from the prey. When $u \neq v$, the term for $\omega_{u v}$ adds more 
1

influence to spiders that are closer and less influence when other spiders are further away; i.e. the weight of influence is inversely proportional to the distance from the spider (the smaller the distance, the greater the influence). To make sure the influence matrix is calculated correctly, the matrix $\omega$ should have the property of

$$
\sum_{k=1}^{n} \omega_{k c}=1
$$

for columns $c \in\{1,2, \ldots, n\}$. The initial level of influence is

$$
\Omega(0)=\left[\begin{array}{cccc}
1 & 0 & \cdots & 0 \\
0 & 1 & \cdots & 0 \\
\vdots & \vdots & \ddots & \vdots \\
0 & 0 & \cdots & 1
\end{array}\right],
$$

the identity matrix. This is under the assumption no spiders are moving initially. We make a note that in the simulations of our model, we assume that all individual spiders have the same motivation to pursue detected prey. There is much empirical evidence to suggest that this is not the case in $A$. studiosus colonies, and that, in fact, individuals have distinct and consistent 'personalities' of aggressiveness across contexts [21]. Furthermore, the composition of personalities in the foraging group are known to affect the overall foraging success of the colonies [18, 19, 20]. Future modifications of our model could incorporate a 'personality parameter' which effects the level of influence to explore the interplay between personality, spacing, and foraging success.

\subsection{Implementation}

Now that we have the perceived location, moving variable, and level of influence, we implement the extended model for multiple spiders into Equation (2). Recall that $\Omega$ and $\Phi$ are the levels of influence and the angles of incidence respectively. Let $\Phi^{\omega}$ be the angles of incidence based on the corresponding levels of influence. Thus,

$$
\Phi^{\omega}=\Omega \cdot \Phi
$$


2

$$
\Phi^{\omega}=\left[\begin{array}{cccc}
* & \omega_{12} & \cdots & \omega_{1 n} \\
\omega_{21} & * & \cdots & \omega_{2 n} \\
\vdots & \vdots & \ddots & \vdots \\
\omega_{n 1} & \omega_{n 2} & \cdots & *
\end{array}\right] \cdot\left[\begin{array}{cccc}
\phi_{1} & \phi_{12} & \cdots & \phi_{1 n} \\
\phi_{21} & \phi_{2} & \cdots & \phi_{2 n} \\
\vdots & \vdots & \ddots & \vdots \\
\phi_{11} & \omega_{12} \phi_{12} & \cdots & \omega_{1 n} \phi_{1 n} \\
\phi_{21} \phi_{21} & \phi_{2} & \cdots & \phi_{n}
\end{array}\right] .
$$

The diagonal entries of $\Phi^{\omega}$ is forced to equal $\phi_{u}$ (Eq. (11)), since this angle of incidence is already accounted for in the range of localization based on the prey's level of influence.

Let's denote $\Theta_{u}$ as the final direction of travel for spider $u$. Assuming the angle of incidence $\phi_{u}$ is independent from the angles of incidence influenced by the other spiders, we take $\phi_{u}$ and add it to the sum of the angles of incidence influenced by the other spiders and divide this by the number of moving spiders. Thus,

$$
\Theta_{u}=\phi_{u}+\frac{1}{r} \sum_{k=1, k \neq u}^{n} \Phi_{u k}^{\omega}
$$

for columns $k \in\{1,2, \ldots, n\}$. Figure 5 shows the final direction in the case of the two spiders given in Figure 4. The thick black arrow and $\Theta_{1}$ and $\Theta_{2}$ indicates the direction and magnitude of the velocity for spider 1 and 2 respectively.

To complete our model incorporating multiple spider interaction, we replace the angle of incidence of Equation (2) by Equation (18). Thus, the extended stochastic differential equation for multiple spiders is given by

$$
\left[\begin{array}{c}
d X_{u}(t) \\
d Y_{u}(t)
\end{array}\right]=v_{u}\left[\begin{array}{c}
\cos \left(\phi_{u}+\frac{1}{r} \sum_{k=1, k \neq u}^{n} \Phi_{u k}^{\omega}\right) \\
\sin \left(\phi_{u}+\frac{1}{r} \sum_{k=1, k \neq u}^{n} \Phi_{u k}^{\omega}\right)
\end{array}\right] d t+\left[\begin{array}{cc}
\sigma_{u} & 0 \\
0 & \sigma_{u}
\end{array}\right] d \mathbf{W}(t)
$$

for spider $u \epsilon\{1,2, \ldots, n\}$ and $n$ is the number of spiders. To simplify,

$$
\left[\begin{array}{l}
d X_{u}(t) \\
d Y_{u}(t)
\end{array}\right]=v_{u}\left[\begin{array}{c}
\cos \left(\Theta_{u}\right) \\
\sin \left(\Theta_{u}\right)
\end{array}\right] d t+\left[\begin{array}{cc}
\sigma_{u} & 0 \\
0 & \sigma_{u}
\end{array}\right] d \mathbf{W}(t)
$$

If $r=1$, then Equation (19) reduces to Equation (2). If $r>1$, then the SDE that governs the predation movement for multiple spiders is Equation (19). 


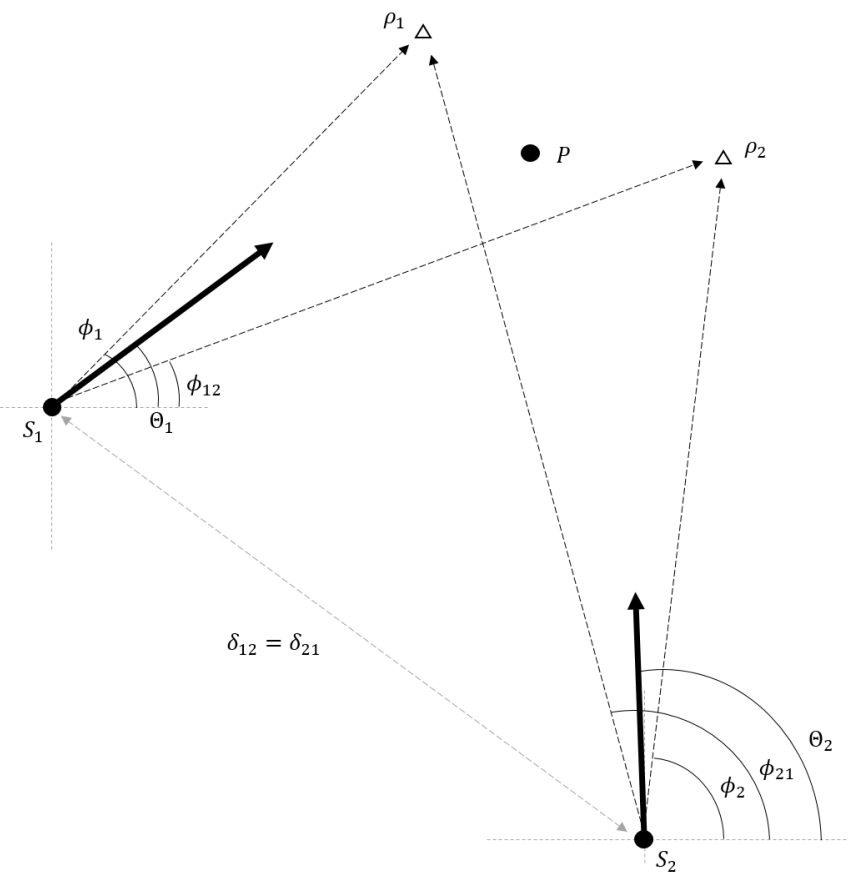

Figure 5: Final Direction Illustration. (see Figure 4)

1 Recall that the algorithm in Figure 1 is the algorithm for one spider. Since 2 we have a model that includes multiple spiders on the web, the algorithm is 3 extended. Figure 6 shows the extended algorithm where $\tau_{u}^{(r)}$ is added after 4 the starting position and $\Theta_{u}$ is added after the second updates.

\section{5. Spatial Distributions of Spider Colony}

$6 \quad$ The main purpose of modeling the predation movement of the A. studio7 sus is to determine if the spiders position themselves accordingly such that 8 they can catch the most prey and avoid potential predators. To determine 9 the optimal spatial distribution of the spiders in the web for prey capture, we model and test different spatial distributions. We define the size of the web to be a square with length $\diamond$ (as discussed in Section 3). We also need 12 a defining threshold of when the spider is considered near the edge. This is 3 denoted by $\hat{\Delta}$. 


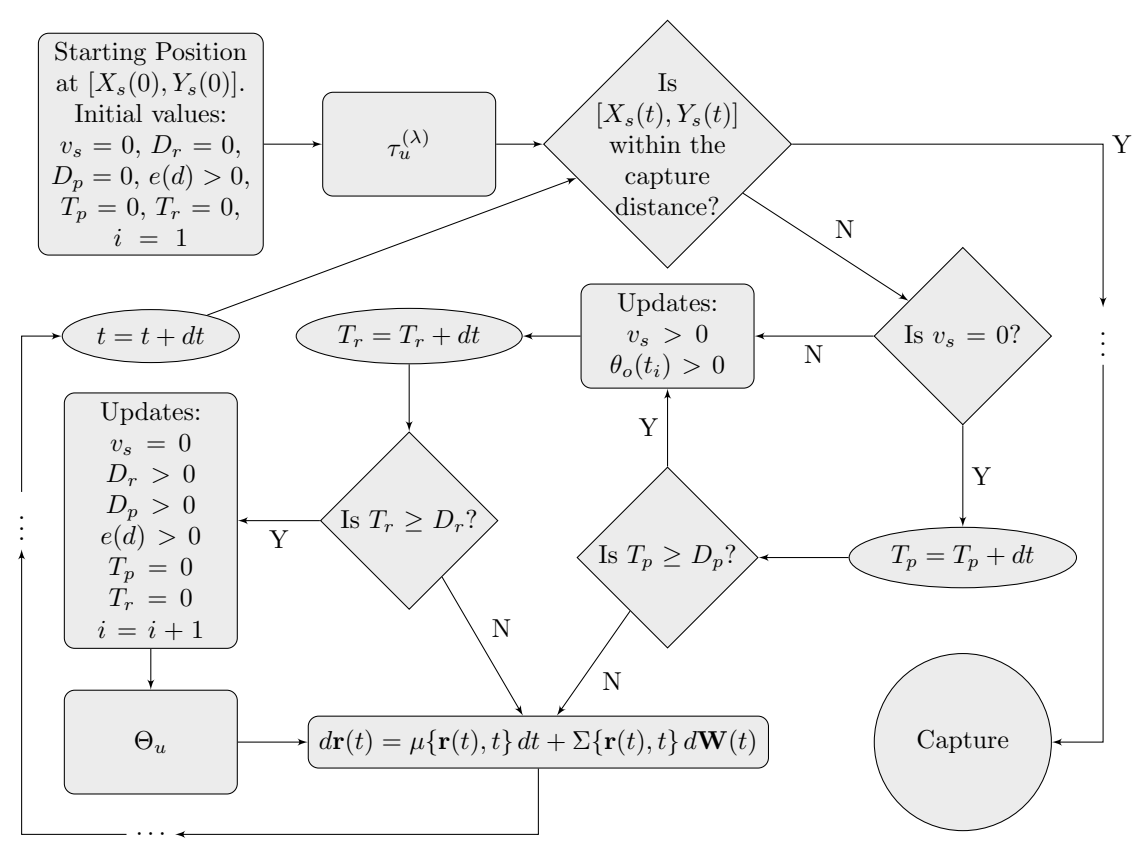

Figure 6: The Stochastic Model Algorithm with Extensions $\tau_{u}^{(r)}$ and $\Theta_{u}$.

11

5

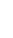

\subsection{Random}

In a random spatial distribution, spiders are randomly placed on the web.

For a web size of $\diamond$, the initial location of spider $u \epsilon\{1,2, \ldots, n\}$ for a random spatial distribution is given by

$$
S_{u}(0)=\left[X_{u}(0) Y_{u}(0)\right] \sim[U(\hat{\diamond}, \diamond) U(\hat{\diamond}, \diamond)]
$$

where $U$ is the uniform distribution. Figure 7 is an example.

\subsection{Edge Random}

We denote the distribution 'Edge Random' when the spiders are randomly placed on the edge of the web. First we take random points from each side of the web. This is calculated by

$$
\bar{E}=\left[\begin{array}{cc}
U(0, \diamond) & U(0, \hat{\diamond}) \\
U(0, \hat{\diamond}) & U(0, \diamond) \\
U(\diamond-\hat{\diamond}, \diamond) & U(0, \diamond) \\
U(0, \diamond) & U(\diamond-\hat{\diamond}, \diamond)
\end{array}\right]
$$




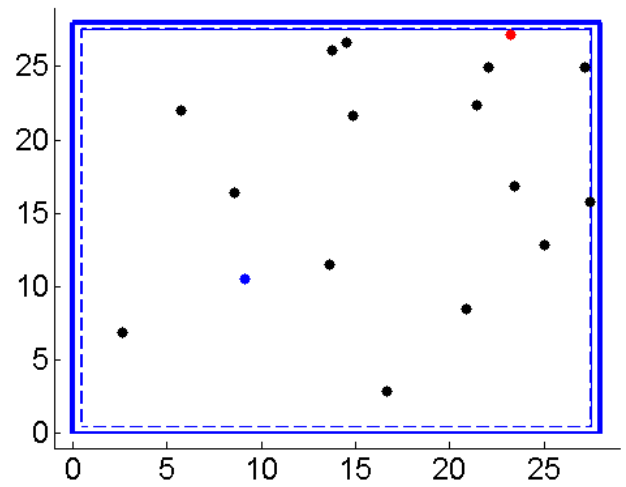

Figure 7: Random Spatial distribution $(n=16)$; Red dot (mother), Black dots (juveniles), and Blue dot (prey).

1 For a web size of $\diamond$, the initial location of spider $u \epsilon\{1,2, \ldots, n\}$ for a edge 2 random spatial distribution is given by

$$
S_{u}(0)=\left[X_{u}(0) Y_{u}(0)\right] \sim \bar{E}_{(U(1,4))}
$$

4 where the subscript of $\bar{E}$ is the row of the matrix $\bar{E}$ taken from a uniform 5 distribution from 1 to 4 . Figure 8 is an example.

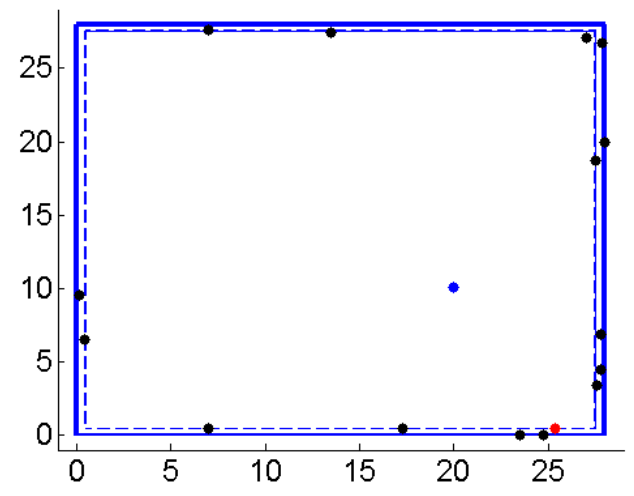

Figure 8: Edge Random Spatial distribution $(n=16)$; Red dot (mother), Black dots (juveniles), and Blue dot (prey).

\section{5.3. Clustered}

7 In a clustered distribution, spiders are randomly placed close to each 8 other at a random reference point. Let $R_{c}$ be the reference point taken from 

$\diamond)$. Figure 9 is an example.

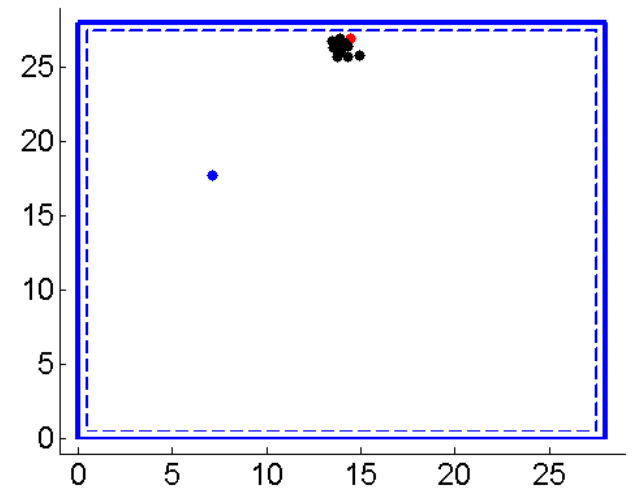

Figure 9: Clustered Spatial distribution $(n=16)$; Red dot (mother), Black dots (juveniles), and Blue dot (prey).

a uniform distribution given by

$$
R_{c}=\left[R_{x} R_{y}\right]_{c} \sim[U(\hat{\diamond}, \diamond-\hat{\diamond}) U(\hat{\diamond}, \diamond-\hat{\diamond})] .
$$

For a web size of $\diamond$, the initial location of spider $u \epsilon\{1,2, \ldots, n\}$ for a clustered spatial distribution is given by

$$
S_{u}(0)=\left[X_{u}(0) Y_{u}(0)\right] \sim\left[N\left(R_{x}, \hat{\diamond}\right) N\left(R_{y}, \hat{\diamond}\right)\right]_{c}
$$

where $N$ is the normal distribution with mean $R_{x}$ and $R_{y}$ for the $x y$ location with a standard deviation of $\hat{\diamond}$. If $R_{c}$ is really close to the edge, this will cause the normal distribution to pick a random point outside of the web. Thus, we truncate the normal distribution if $R_{c}$ is really close to the edge (i.e. 0 and

\subsection{Edge Clustered}

We define 'Edge Clustered' to be the clustered distribution in which the reference point is on the edge of the web. First we randomly pick a point from the edges similar to the Edge Random, and we pick a random point from those 4 random points. This is given by

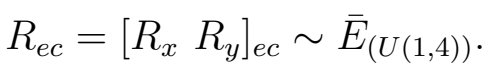

where the subscript of $\bar{E}$ is the row of the matrix $\bar{E}$ taken from a uniform distribution from 1 to 4 . For a web size of $\diamond$, the initial location of spider $u \in\{1,2, \ldots, n\}$ for a edge clustered spatial distribution is given by

$$
S_{u}(0)=\left[X_{u}(0) Y_{u}(0)\right] \sim\left[N\left(R_{x}, \hat{\diamond}\right) N\left(R_{y}, \hat{\diamond}\right)\right]_{e c}
$$


1 where $N$ is the normal distribution with mean $R_{x}$ and $R_{y}$ for the $x y$ location 2 with a standard deviation of $\hat{\diamond}$. Since the $R_{e c}$ is at the edge, this will induce 3 the normal distribution to pick a random point outside of the web. Thus, we 4 truncate the normal distribution if $R_{e c}$ is really close to the edge (i.e. 0 and $5 \diamond)$. Figure 10 is an example.

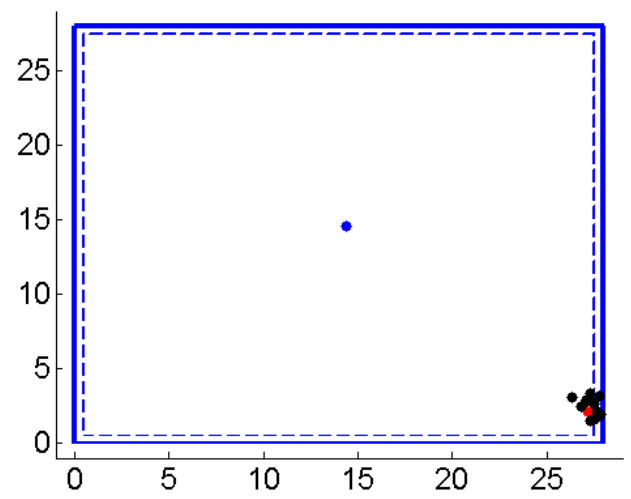

Figure 10: Edge Clustered Spatial distribution $(n=16)$; Red dot (mother), Black dots (juveniles), and Blue dot (prey).

\subsection{Uniform}

In a uniform distribution, the spiders are located on the web in a gridlike pattern. Let $\aleph$ be the number of rows and columns of the grid given by $\aleph=\left[\frac{n}{\sqrt{n}}\right]$ for $n \epsilon\{4,9,16,25, \ldots\}$. Let $A$ and $B$ be vectors of possible $x$ and $y$ values of each spider given by$$
A_{i}=B_{i}=\frac{\diamond i}{\aleph+1}, i \epsilon\{1, \ldots, \aleph\} .
$$

The location of spider $u \epsilon\{1,2, \ldots, n\}$ is given by

$$
S_{u}(0)=\left[X_{u}(0) Y_{u}(0)\right]=\left[A_{i} B_{j}\right]
$$

for $i, j \in\{1, \ldots, \aleph\}$. Figure 11 is an example.

\subsection{Edge Uniform}

'Edge Uniform' spatial distribution is where the spiders are placed uniformly along the edge. To place the $n$ spiders on a $\diamond \times \diamond$ web, we take $d=P / n$ 


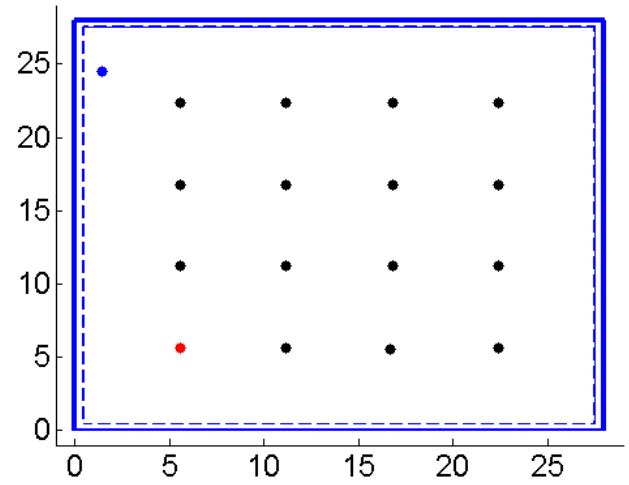

Figure 11: Uniform Spatial distribution $(n=16)$; Red dot (mother), Black dots (juveniles), and Blue dot (prey).

where $P$ is the perimeter of the square, $d$ is the distance between successive spiders, $\diamond$ is the length of the sides, $\hat{\diamond}$ is the edge threshold, and $\mathrm{n}$ is the number of spiders. Note that we want the spider in between the threshold. So, the perimeter should have $P=\diamond-2(\hat{\diamond} / 2)$. Therefore, $d=\frac{\vartheta-\hat{\vartheta}}{n}$.

If we label the edges as $s_{1}$ (bottom edge), $s_{2}$ (right edge), $s_{3}$ (top edge), and $s_{4}$ (left edge), then we place the first spider at the center of side 1 . We force the first spider to be at the center instead of placing it randomly on side 1 to induce symmetric displacement of the spiders about a vertical line at the center of the square. After the first spider is placed, we place the next spider to the right at a distance of $d$. If the next spider has a distance $\hat{d}<d$ where $\hat{d}$ is the distance from the previous spider to the corner, then the next spider goes on to the next side with a distance $d-\hat{d}$ from the corner. Thus, the location of spider $u \in\{1,2, \cdots, n\}$ is given by

$$
X_{u}(0)=\left(X_{u}(0), Y_{u}(0)\right)=\left\{\begin{array}{cc}
(\diamond / 2, \hat{\diamond} / 2), & u=1 \\
(\hat{\diamond} / 2+(d-\hat{d}), \hat{\diamond} / 2), & \hat{d}<d, s_{4} \rightarrow s_{1} \\
\left(X_{u-1}+d, \hat{\diamond} / 2\right), & s_{1} \\
(\diamond-\hat{\diamond} / 2, \hat{\diamond} / 2+(d-\hat{d})), & \hat{d}<d, s_{1} \rightarrow s_{2} \\
\left(\diamond-\hat{\diamond} / 2, X_{u-1}+d\right), & s_{2} \\
(\diamond-\hat{\diamond} / 2-(\diamond-\hat{\diamond}), \diamond-\hat{\diamond} / 2), & \hat{d}<d, s_{2} \rightarrow s_{3} \\
\left(X_{u-1}-d, \diamond-\hat{\diamond} / 2\right), & s_{3} \\
(\hat{\diamond} / 2, \diamond-\hat{\diamond} / 2-(\diamond-\hat{\diamond})), & \hat{d}<d, s_{4} \rightarrow s_{1} \\
\left(\hat{\diamond} / 2, X_{u-1}-d\right), & s_{4}
\end{array}\right.
$$

Since we started at the center of side 1 , then the $n$th spider should be at a 
distance of $d$ to the left of the first spider.

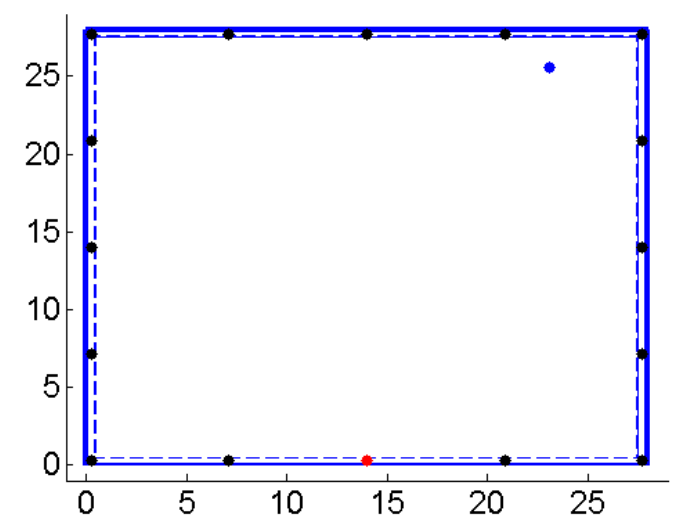

Figure 12: Edge Uniform Spatial Distribution $(\mathrm{n}=16)$

2

3

\subsection{Grouped}

'Grouped' is defined by the spiders being distributed in groups, where a group is defined as a certain number spiders who are close together. If a spider is by itself but separate to a group of spiders, then it is also considered a group of one spider. Let $G$ be the number of groups given by

$$
G \sim U(\{2,4\})
$$

where $U(\{2,4\})$ is a uniform distribution with sample space $\{2,4\}$. We want to find $G$ number of reference points for $G$ groups. Let $R_{g}$ be a matrix containing $G$ reference points which is given by

$$
R_{g}=\left[R_{x}(0) R_{y}(0)\right]_{g}=[U(\hat{\diamond}, \diamond-\hat{\diamond}) U(\hat{\diamond}, \diamond-\hat{\diamond})]
$$

for $g \in\{2, \ldots, G\}$. Each group should be far enough from each other to be considered separate groups. Suppose we have $G$ groups, then the sum of the number of spider is

$$
\sum_{g=1}^{G} \hat{s}_{g}=\hat{s}_{1}+\hat{s}_{2}+\cdots+\hat{s}_{g}=n
$$

where $\hat{s}_{g}$ is the number of spiders of group $g$. The total number of spiders is randomly distributed among $G$ groups. This is given by

$$
\hat{s}_{g}=U\left(1,\left\lceil\frac{n}{g}\right\rceil\right) \text {. }
$$



$\diamond)$. Figure 13 is an example.

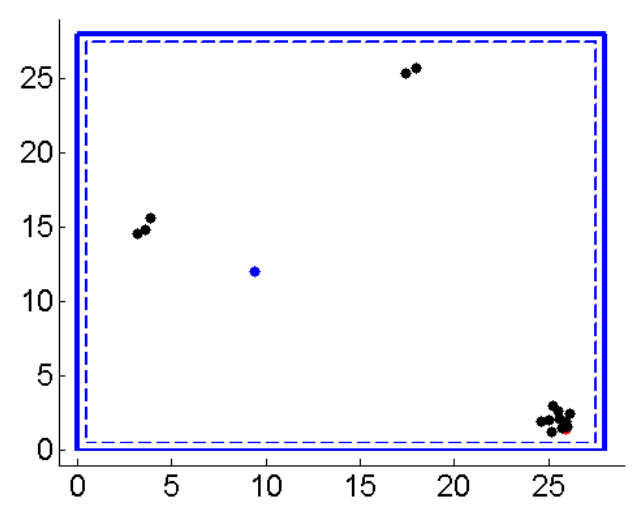

Figure 13: Grouped Spatial distribution $(n=16)$; Red dot (mother), Black dots (juveniles), and Blue dot (prey).

\subsection{Edge Grouped}

$$
S_{k}(0)=\left[X_{k}(0) Y_{k}(0)\right] \sim\left[N\left(R_{g}, \hat{\diamond}\right) N\left(R_{g}, \hat{\diamond}\right)\right]
$$

where $k \epsilon\left\{1, \ldots, \hat{s}_{g}\right\}$. If $R_{g}$ is really close to the edge, this will cause the normal distribution to pick a random point outside of the web. Thus, we truncate the normal distribution if $R_{g}$ is really close to the edge (i.e. 0 and

'Edge Grouped' is where the spiders are distributed in groups along the edge. The method to randomly place the spider on the web in this kind of distribution is the same method as grouped, but we randomly pick reference points from the edges. First we randomly pick a point from the edges similar to the Edge Random and we pick a random point from those 4 random points. This is given by

$$
R_{e g}=\left[\begin{array}{ll}
R_{x} & R_{y}
\end{array}\right]_{e g} \sim \bar{E}_{(U(1,4))} .
$$

where the subscript of $\bar{E}$ is the row of the matrix $\bar{E}$ taken from a uniform distribution from 1 to 4 . Given $R_{g}$ and $\hat{s}_{g}$ (see Grouped section), then the location of the spiders for each group is given by

$$
S_{k}(0)=\left[X_{k}(0) Y_{k}(0)\right] \sim\left[N\left(R_{e g}, \hat{\diamond}\right) N\left(R_{e g}, \hat{\diamond}\right)\right]
$$

where $k \epsilon\left\{1, \ldots, \hat{s}_{g}\right\}$. If $R_{e g}$ is really close to the edge, this will cause the normal distribution to pick a random point outside of the web. Thus, we truncate the normal distribution if $R_{e g}$ is really close to the edge (i.e. 0 and $\diamond)$. Figure 14 is an example.

Given $R_{g}$ and $\hat{s}_{g}$, then the location of the spiders for each group is given by 


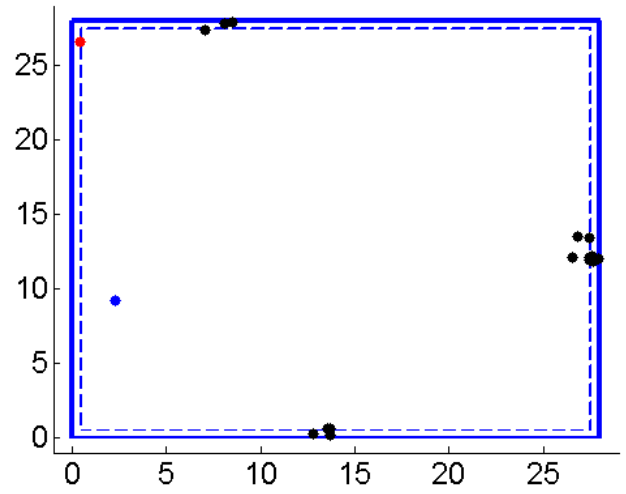

Figure 14: Edge Grouped Spatial distribution $(n=16)$; Red dot (mother), Black dots (juveniles), and Blue dot (prey).

\section{Simulation Results}

Using the spacial distributions from the previous section and the new model for multiple spiders, we want to determine the success rate for prey capture given a particular spatial distribution. For simulation purposes, we assume a square web with dimension $\diamond=28$ and initial location of the prey chosen from a uniform distribution, i.e.

$$
S_{p}(0)=\left[X_{p}(0) Y_{p}(0)\right] \sim[U(\hat{\diamond}, \diamond) U(\hat{\diamond}, \diamond)]
$$

where $U$ is the uniform distribution. We consider four different spider colonies consisting of 4, 9, 16, and 25 spiders. (These size colonies allow a complete grid for the uniform spatial distribution.) We consider both the perceptive and careless prey with varying levels of influence, $0<\omega_{p}<1$ incremented in steps of 0.1 .

Given 4 different colony sizes, 7 spatial distributions, 2 prey phenotype, and 11 levels of influences, there are 616 combinations of these parameters. We perform 1000 simulations for each combination and record the proportion of wins (the spider captures the prey), losses (the prey reaches the edge of the web and escapes), and neither (the spiders cannot find the prey or have no motivation to pursue the prey after 2 minutes). As a note, the case in which the spider doesn't pursue the prey is also evidenced in the experimental lab; therefore, this result is consistent with experimental trials. In total, there are 616,000 simulations to perform. We only summarize the results in this section. 
In Figures 15 - 22, there are two plots, one for the proportion of spider wins and one for the mean time required to capture the prey both as a function of the level of influence of the prey $\omega_{p}$. In each plot, there are eight curves which denote four different colony sizes for each type of prey (perceptive versus careless). As one would expect, the proportion of spider wins increase as the level of influence of the prey gets closer to 1 . Recall the level of influence $\omega_{p}$ determines how strongly the spiders sense the prey. The stronger the influence of prey, the more likely the spiders will find the prey and catch it regardless of prey type. Moreover, as $\omega_{p}$ approaches 1 , the situation in which the prey doesn't locate the edge and the spider doesn't pursue the prey decreases to zero. When $\omega_{p}=0$, spiders who won basically found and caught the prey by random chance. Similarly, in almost all cases, the chances of winning increases as the colony size increases, regardless of prey type.

We take a closer look at the edge random spatial distribution in Figure 16. One question which is interesting to consider is how lucky the spiders can be in capturing the prey if they simply position themselves randomly at the edge but have no sense for where the prey is located $\left(\omega_{p}=0\right)$. Figure 16 (a) shows that there is a $30 \%$ chance of winning at maximum colony size of $n=25$ even though $\omega_{p}=0$ and the prey is perceptive. This is higher than any other spatial distribution where the maximum chance of capturing the prey by simple luck is typically less than $10 \%$ with the exception being when the spiders are grouped on the edge (see Figure 22). Also, in the case of random edge distribution (Figure 16), it takes only $30-50$ seconds to catch the prey by 'luck'. This range is lower than the other spatial distributions indicating they are able to capture the prey faster even when they have no influence from the prey itself. We can also discern from Figure 16 that both the mean time elapsed and proportion of wins appear to approach an equilibrium as $\omega_{p}$ approaches 1 . When $\omega_{p}>0.6$, the benefit of being able to better sense the prey, i.e. increase $\omega_{p}$, has less increase in impact on the overall success in capture. One other pattern which is distinguishable is that the mean time elapsed has a discernible pattern where the time elapsed is increasing beginning at $\omega_{p}=0$ and decreasing around $\omega_{p}=0.2$. One would expect simply a decrease in time as $\omega_{p}$ gets larger; therefore, we are uncertain of the meaning for this discernable pattern.

Our ultimate goal was to determine which spatial distribution works best for the spiders during predation. Figure 23 shows the proportion of wins for each distribution, level of prey influence and colony size. Red indicates 


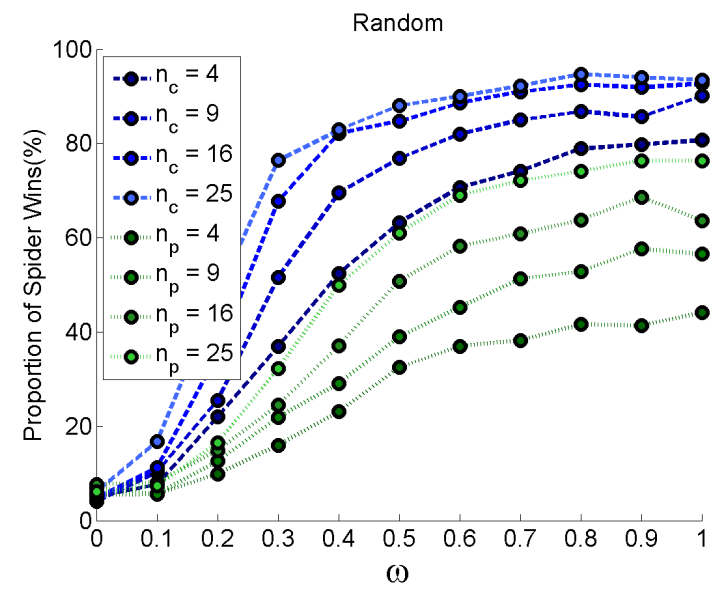

(a) Proportion of Spider Wins (\%)

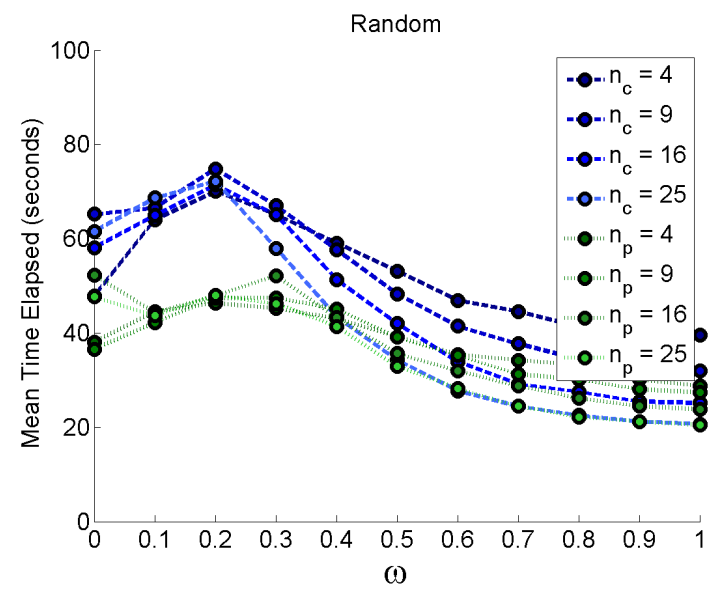

(b) Mean Time Elapsed (seconds)

Figure 15: Results with random spatial distribution with four colony sizes. The blue and green lines indicates careless and perceptive prey respectively. (ex. $n_{c}=4$ means colony of size 4 with careless prey.)

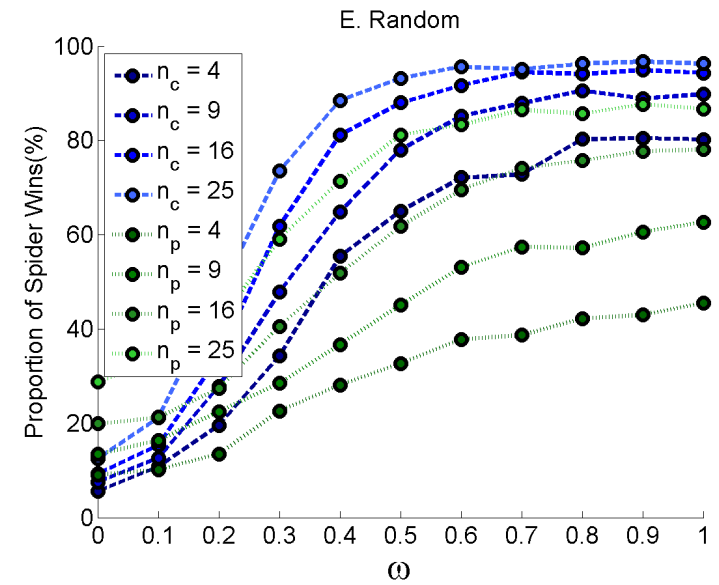

(a) Proportion of Spider Wins (\%)

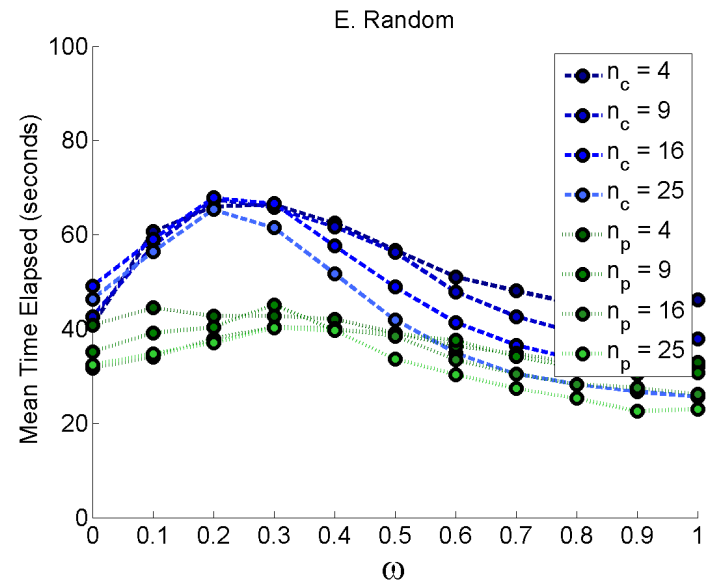

(b) Mean Time Elapsed (seconds)

Figure 16: Results with edge random spatial distribution with four colony sizes. The blue and green lines indicates careless and perceptive prey respectively. (ex. $n_{c}=4$ means colony of size 4 with careless prey.)

1 a higher success rate. There is an obvious difference between the careless 2 and perceptive prey groups. Naturally, the proportion of spider wins with 3 perceptive prey is lower than the proportions with careless prey, because the 


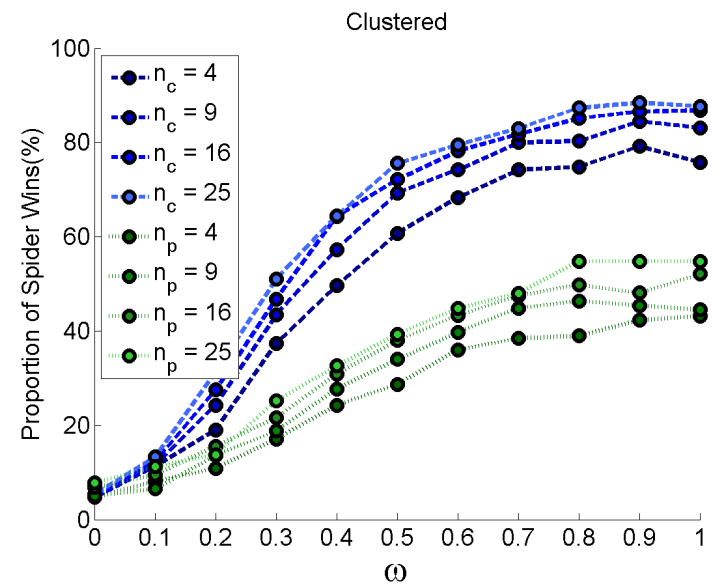

(a) Proportion of Spider Wins (\%)

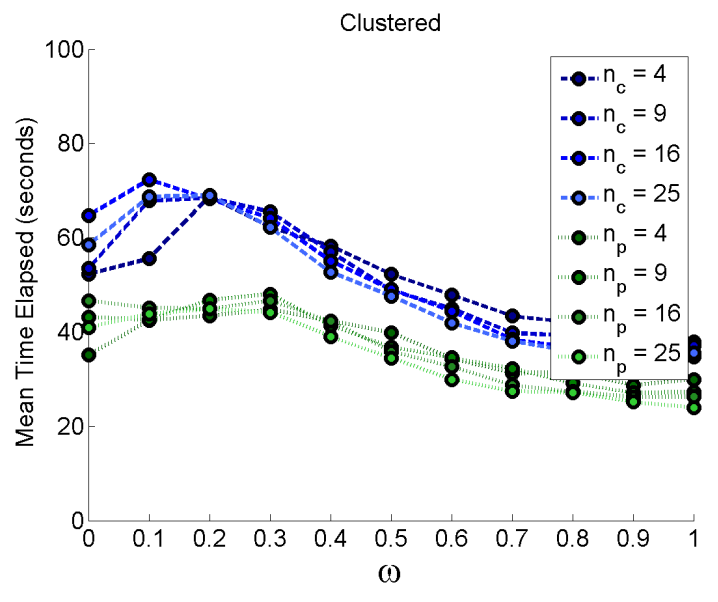

(b) Mean Time Elapsed (seconds)

Figure 17: Results with clustered spatial distribution with four colony sizes. The blue and green lines indicates careless and perceptive prey respectively. (ex. $n_{c}=4$ means colony of size 4 with careless prey.)

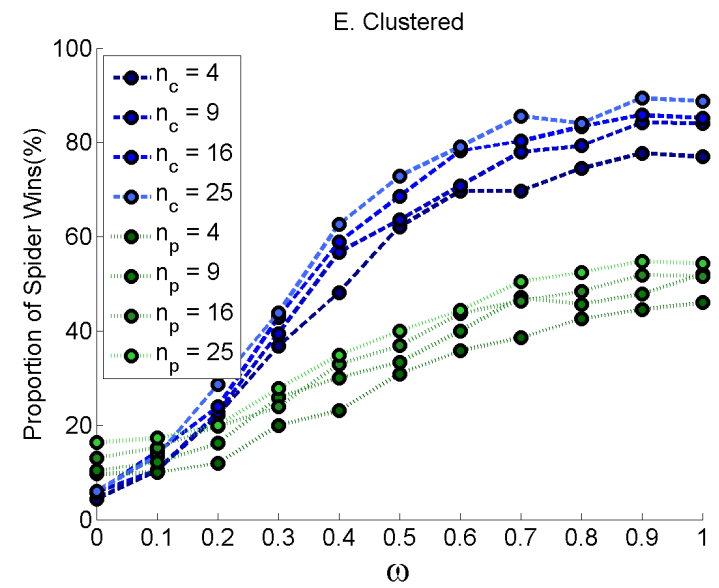

(a) Proportion of Spider Wins (\%)

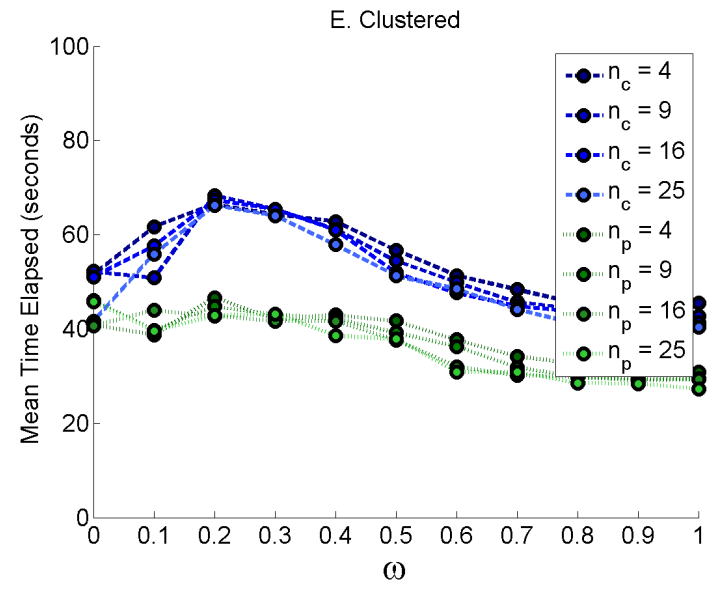

(b) Mean Time Elapsed (seconds)

Figure 18: Results with edge clustered spatial distribution with four colony sizes. The blue and green lines indicates careless and perceptive prey respectively. (ex. $n_{c}=4$ means colony of size 4 with careless prey.)

1 perceptive prey immediately tries to escape to the nearest edge. This is where 2 the comparison between two prey type is very important in determining 3 which spatial distribution works best for the spiders, because regardless of 


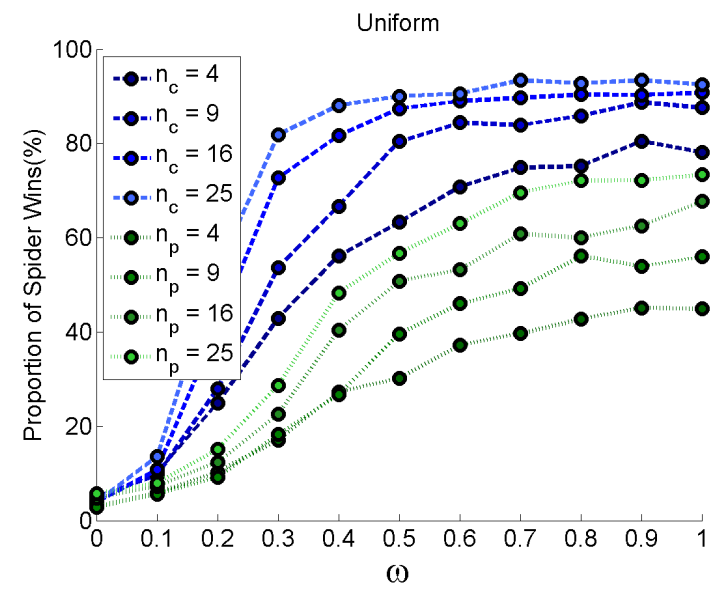

(a) Proportion of Spider Wins (\%)

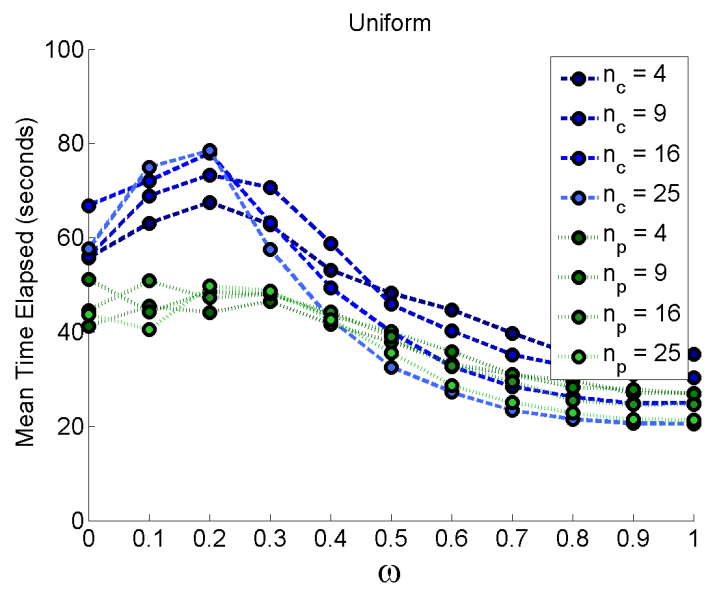

(b) Mean Time Elapsed (seconds)

Figure 19: Results with uniform spatial distribution with four colony sizes. The blue and green lines indicates careless and perceptive prey respectively. (ex. $n_{c}=4$ means colony of size 4 with careless prey.)

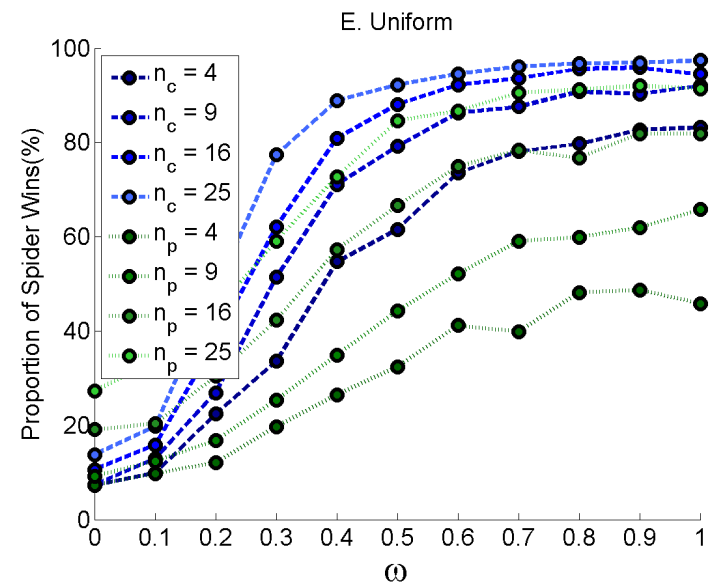

(a) Proportion of Spider Wins (\%)

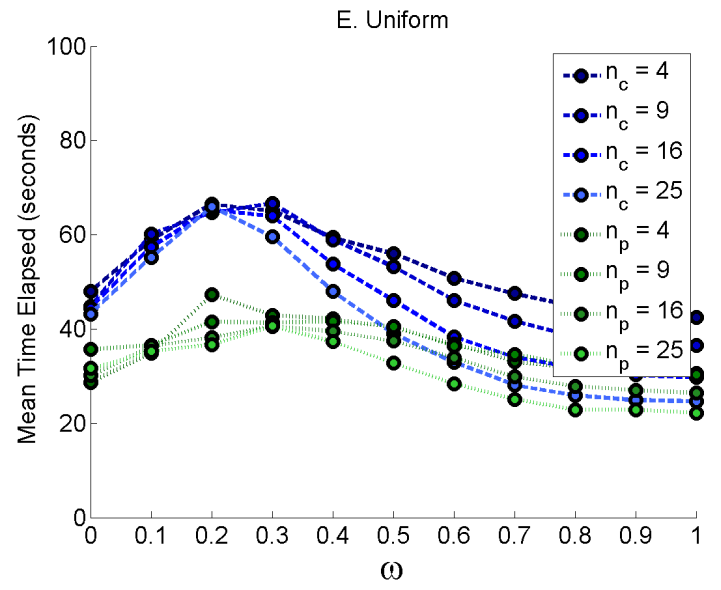

(b) Mean Time Elapsed (seconds)

Figure 20: Results with edge uniform spatial distribution with four colony sizes. The blue and green lines indicates careless and perceptive prey respectively. (ex. $n_{c}=4$ means colony of size 4 with careless prey.)

1 prey type the spiders need to catch as many prey as possible. If one focuses 2 on a single level of influence, say $\omega_{p}=0.6$, the best success rate happens 3 when the spiders are positioned uniformly on the edge followed closely by the 


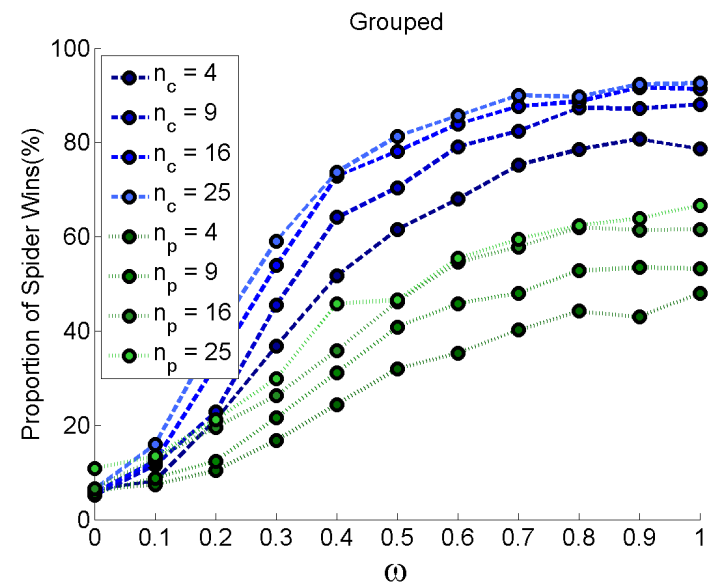

(a) Proportion of Spider Wins (\%)

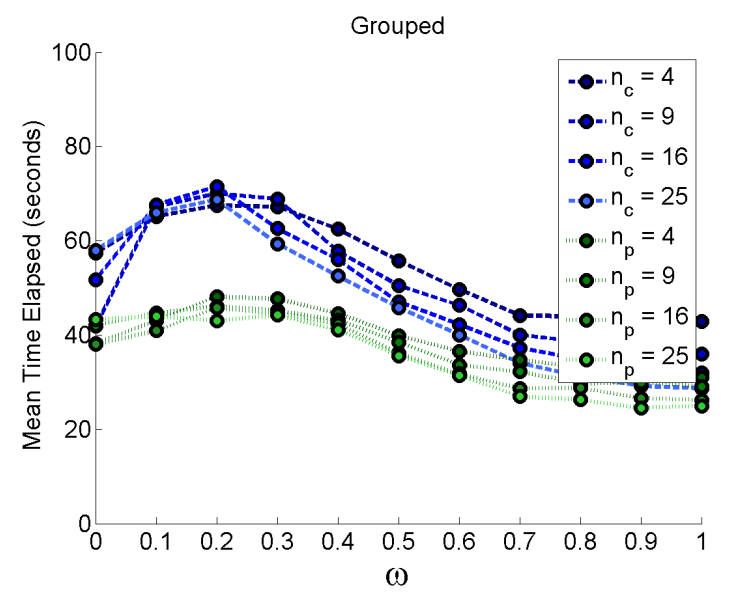

(b) Mean Time Elapsed (seconds)

Figure 21: Results with edge grouped spatial distribution with four colony sizes. The blue and green lines indicates careless and perceptive prey respectively. (ex. $n_{c}=4$ means colony of size 4 with careless prey.)

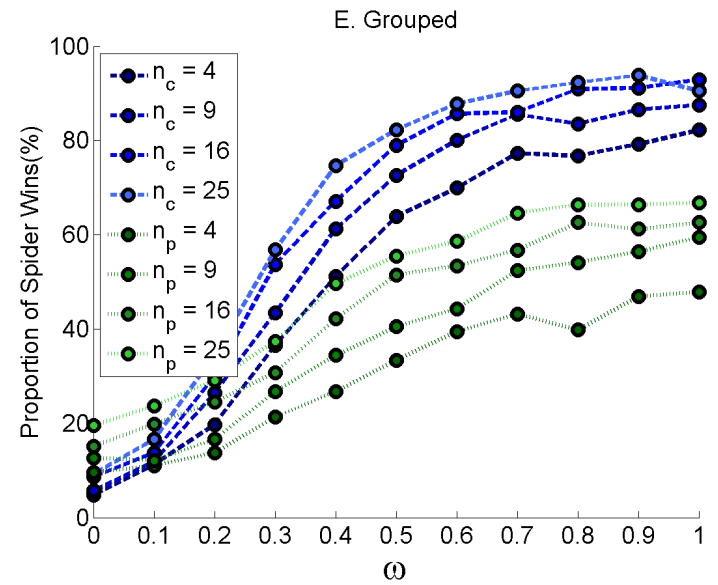

(a) Proportion of Spider Wins (\%)

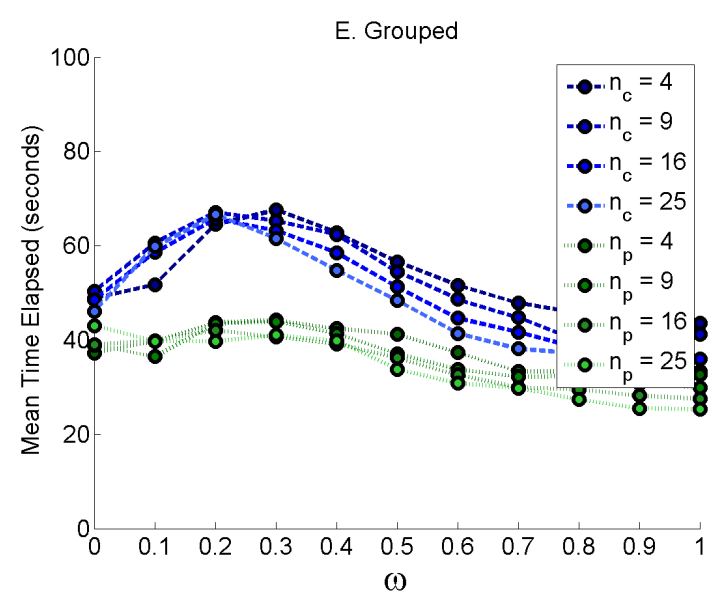

(b) Mean Time Elapsed (seconds)

Figure 22: Results with edge grouped spatial distribution with four colony sizes. The blue and green lines indicates careless and perceptive prey respectively. (ex. $n_{c}=4$ means colony of size 4 with careless prey.)

1 spiders positioned randomly on the edge. This makes sense since typically 2 prey cannot fly out of the web and must move to an edge to escape. 
Proportion of -Spider Wins- (\%) with Careless Prey

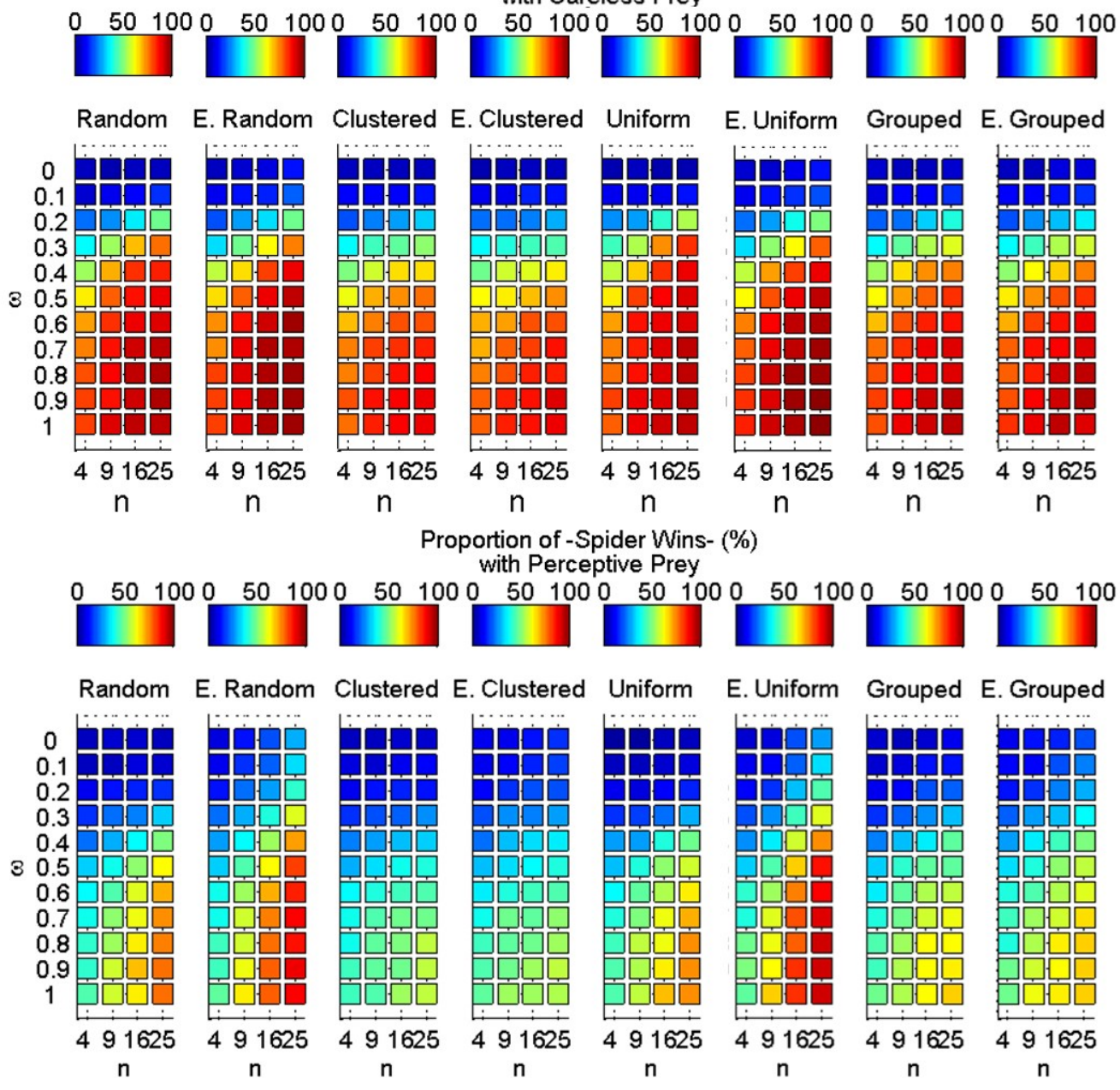

Figure 23: Proportion of Wins 


\section{Experimental Results}

In the thesis by Ross [25], data is presented on the variation of the position of $A$. studiosus in the web throughout the daily cycle. Considering the fact that this species cooperatively forages, they hypothesized that there existed some optimal spatial arrangement of mother and spiderlings in the web that maximizes the amount of prey captured. It was found that the mother and spiderlings do position themselves differently throughout the web both as a function of the age of the juvenile as well as a function of the time of day. At certain times during the day, the spiderlings were spaced closer to the edge than what would be expected under complete spatial randomness. During this same time of day, they were farther from their neighbor than at other times of the day. This observation, together with the model simulations in this paper, appear to support the hypothesis that the spiders are positioning themselves for optimal prey capture during certain intervals of the day. In this section, we summarize the experimental results; for a detailed analysis, we refer the reader to [25].

\subsection{Methods}

For this study, A. studiosus females with egg cases were collected at the end of May 2012 at Fort Patrick Henry Lake in northeast Tennessee $\left(36^{\circ} 30 \mathrm{~N}\right.$; $\left.82^{\circ} 28 \mathrm{~W}\right)$. These spiders with their egg cases were placed in $16 \times 16 \mathrm{~cm}$ square, transparent, closed containers of $3 \mathrm{~cm}$ depth. Hair nets were stretched across the openings of these containers to provide a substrate on which the spiders could build their webs. The shape of these containers caused to spiders to build compressed, nearly two dimensional webs, essentially simplifying the webs to only capture sheets as no debris was provided for the spiders to use as a retreat. Spiders were maintained in the lab on a 12:12 light/dark cycle with lights on at 08:00 and lights off at 20:00. They were fed a mixture of live termites, crickets, and drosophila twice a week. Water was provided by misting the webs three times a week.

Once the juveniles emerged and reached the third instar (known to be when they become active in the colony $[5,12])$, their positions in the webs were manually plotted by placing the containers over a grid and plotting one point for the abdomen of each spiderling on a copy of the grid. The position of the mother was specifically noted. Ten colonies were used and the positions of all the spiders in the web were plotted every four hours starting at 03:00 for 24 hours. Dark observations were made under deep 
1

red light (invisible to spiders [8]) so as not to disrupt any diel or circadian rhythms the spiders might have. A total of four observation days were used: one when the spiderlings reached the 3rd instar, one at the end of the growing season, and two in between as dictated by development. Points were digitized using WebPlot Digitizer software [24]; see the left plot in Figure 24 (the red point indicates the position of the mother). This provided $x-y$ coordinates of a high degree of accuracy that could be imported into Excel, MATLAB, MINITAB, and R.

\subsection{Results}

To analyze the spiders' spatial point patterns, three main measures were considered: distance to the nearest neighbor [4], distance to the nearest edge [13], and the percent area of the container the convex hull occupied [4] (a fourth measure, distance to mom, can be found in [25]). Readers who have worked with spatial point patterns will be familiar with distance to the nearest neighbor, in which for each point, the distance to all other points is found, and the smallest of these distances is taken to be the distance to the nearest neighbor. The empirical function of cumulative relative frequencies of distances to the nearest neighbor can be compared to the $G$ function [4] which represents what the cumulative relative frequency curve for distance to the nearest neighbor should look like under complete spatial randomness (CSR). An example is given in the middle plot of Figure 24. As the juveniles aged, it was observed that they transitioned from clustering around mom near the center of the web to showing a preference for the edges of the container. The $B$ function [13] is similar to the $G$ function which is used to analyze the distance to the nearest edge. It is the expected cumulative relative frequency curve of the distance of each point to the nearest edge under CSR. Thus, an empirical $B$ function can be compared to the theoretical $B$ function to help detect any deviations in spatial point patterns from CSR. An example is given in the right plot of Figure 24 which indicates in this example that the spiders are closer to the edge than what would be expected under CSR. The convex hull is a commonly used algorithm that chooses the points which form a polygon that is an effective perimeter of the points such that all points lie within or on the convex hull ([4]). The convex hull is commonly thought of by taking an elastic band and stretching it around a set of fixed points, then releasing the band. When the band comes to rest stretched taught around the points, this is the convex hull of the set. An example is shown in the left 

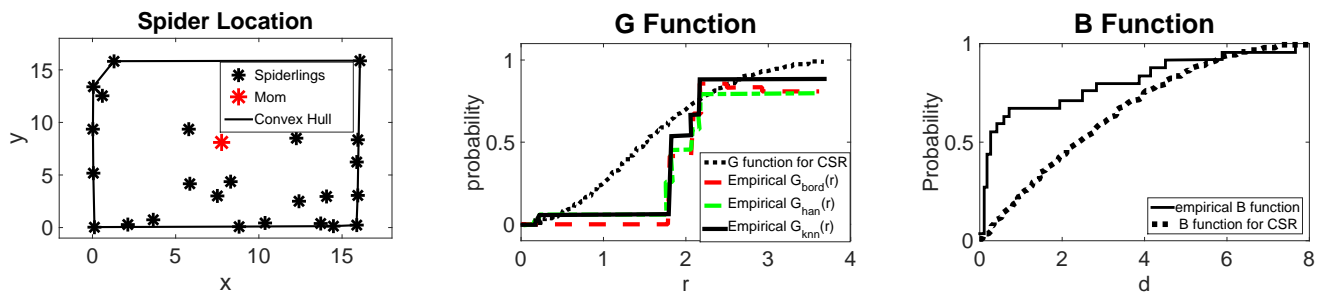

Figure 24: An example of the spatial point pattern at one observation time for one colony (at instar 5) along with the $G$ and $B$ function utilized in a portion of this point pattern analysis

plot of Figure 24. The area of the convex hull was taken as a percentage of the total area available to the spiders $\left(256 \mathrm{~cm}^{2}\right)$.

To calculate these measurements for each colony at each time and day, an $R$ program written by the authors using the packages spatstat and plotrix was used on the raw, digitized data. This was done for each observation, of which there were 240 total (10 colonies, 6 times per day, 4 days). These numbers were then imported into MINITAB [16] for basic statistical analysis. The means and medians of each of these measures over time of day and day of development were examined; the trends are shown in Figure 25. The solid lines represent the colonies without mom while the dashed line indicates those colonies where mom is still present. We note there is a change in all three measurements across the course of the day. Colonies without mom always cover a larger percentage of the entire web (indicated by the percent coverage of the convex hull) than those colonies with mom. This coincides with the graph given in the second row, Figure 25, in which the colonies with mom are always closer to the edge. Furthermore, when analyzing the colonies with mom, as the median distance to the edge decreases (get closer to the edge), the median distance to the nearest neighbor appears to increase (more spread out). This potentially could be the spiders spreading out along the edge at certain times of the day. When analyzing changes as a function of the day of development, we see that for colonies without mom, there is very little change in any of the measurements; however, in colonies with mom, spiders tend to be farther from their neighbor, closer to the edge and cover a larger portion of the total area on day 48 than day 6 . Further analysis indicated that on day 48 , there was a statistically significant preference for the spiderlings to be within $1 \mathrm{~cm}$ of the edge as opposed to the interior in a 

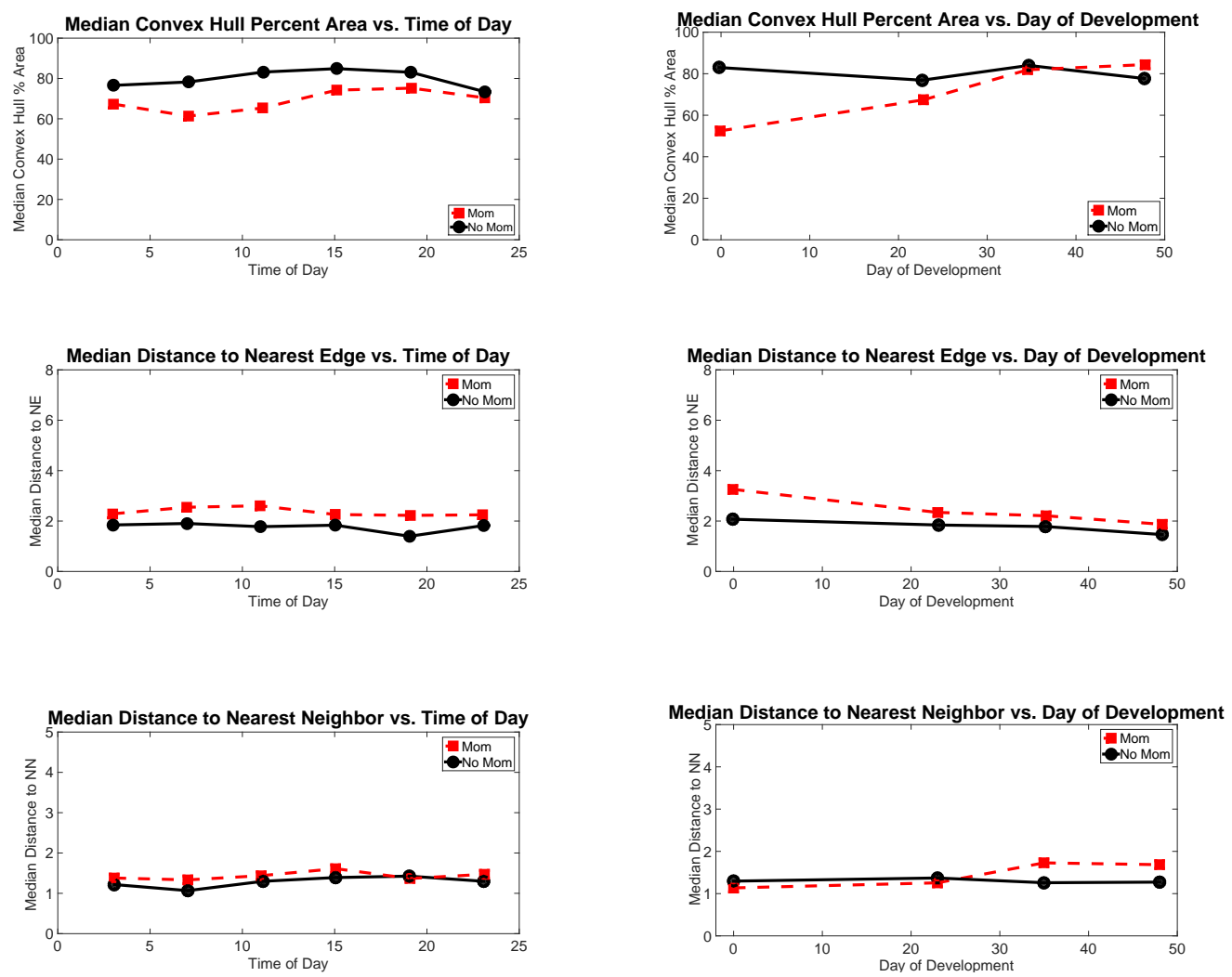

Figure 25: Medians of convex hull percent area, distance to nearest neighbor and distance to nearest edge shown over time of day and development. Colonies with mom are shown in red while colonies without mom are shown in black

majority of the observations.

\section{Conclusions}

In conclusion, we adapted a model by Joyner et. al. [2] and Quijano et.al. [22] for the movement of the subsocial spider Anelosimus studiosus during predation to include the effects of interaction among spiders in the web while foraging. The interaction took into account the level of influence from the prey as well as the influence from other spiders within the web. Moreover, the model was further adapted to include a latency period exhibited by Anelosimus studiosus experimentally. The model was then used to test multiple spatial configurations to determine which spatial configuration 
was the most effective for the colony for foraging. Based on the simulations, the best spatial distribution was an edge uniform distribution in which the spiders spaced out uniformly along the edges of the web. However, spiders spacing out randomly along the edge was a close second in terms of the best spatial configuration. This is similar to the behavior observed by Ross [25] and summarized in Section 7 in which it was determined to be statistically significant that during certain times of the day, spiders were positioned along the edge more than expected under complete spatial randomness. Therefore, one might hypothesize that during this time of the day, the spiders might be positioning themselves to be most successful for foraging.

Cooperative hunting can be enhanced if individuals which detect prey signal others to join in the hunt [29]. In most studies, the signal is a display or call specifically communicating to other members of the group to alert them to the presence of prey $[10,27,28]$. However, the signal may not be a specific communication behavior, rather a consequence of the individuals hunting behavior drawing the attention of others in the group. Such could be the case during the final death chase observed in A. studiosus $[2,22]$ in which the prey elevates its escape behavior in proximity to the approaching spider. This increased activity of both spider and prey would likely alert other spiders across a greater expanse of the web. Such recruitment of social spiders to prey has been observed in a social congener Anelosimus eximius, where larger prey grasped by a spider recruit spiders from farther away in the web [17]. However, an interesting caution to the limits of increasing effectiveness with group size may be demonstrated by groups of foraging bats [7]. In this case the ability of bats to find and track prey was enhanced by foraging in mediumsized groups, and bats could aggregate by eavesdropping on other foraging bats (rather than detecting the prey itself). At high bat densities, however, foraging efficiency was reduced by the echolocation calls masking the prey reflections, and by bats having to track and avoid other bats. Our spider system may follow a similar pattern where the increased vibrations of the 'death chase' could draw other spiders to the general location of prey in the web, but make differentiating prey from other spiders more difficult. In the future, the tractable nature of social spider colonies would facilitate empirical studies of detection and coordination in cooperatively foraging animals.

Not surprisingly, our simulations found that larger numbers of spiders are more efficient at capturing prey over the same area. However, in $A$. studiosus the size of the web correlates very tightly with the number of spiders in the colony [26], and in other social spiders reduction in energetic 
costs of web maintenance are identified as one the advantages of sociality [15]. Presumably larger webs intercept more prey, but, as shown here, lower densities of spiders are less likely to capture intercepted prey. In future work we will build on this current model exploring how the optimal web size changes with the number of hunting spiders.

Cooperative hunting can be enhanced if individuals can coordinate their efforts such as is observed in African wild dogs [8]. Other models suggest that collective foraging can be enhanced without complex or specific communication among foragers [28] particularly by increasing the collection prey perception by the group [3]. Our model changes in prey capture efficiency associated with different spatial distributions of spiders in a communal web. This leads to questions and speculation as to if, and how, the spiders could coordinate such distributions. Presumably individual spiders can detect the edge of the web and wait there for prey. However, A. studiosus, and other web-building spiders, have relatively poor vision [1] and could not likely see other webmates well enough to coordinate their spacing. Likely they would have to rely on sensing the vibrations of other spiders moving in the web nearby to judge their proximity and move appropriately. In the future we will analyze individuals movements in the web to detect if they are using their limited information to optimize spacing in the web.

\section{Acknowledgements}

This research was partially supported by the NSF grant DMS-1128954. We would also like to acknowledge Nathaniel Hancock and Michael Largent for their efforts in the collection of the data used for this project. 


\section{References}

[1] Ingi Agnarsson, Wayne P Maddison, and Leticia Avilés. The phylogeny of the social anelosimus spiders (araneae: Theridiidae) inferred from six molecular loci and morphology. Molecular phylogenetics and evolution, 43(3):833-851, 2007.

[2] Michele L. Joyner andChelsea Ross, Colton Watts, and Thomas C. Jones. A stochastic simulation model for Anelosimus Studiosus during prey capture: A case study for determination of optimal spacing. Mathematical Biosciences and Engineering, 11:1411-1429, 2014.

[3] Andrew Berdahl, Colin J Torney, Christos C Ioannou, Jolyon J Faria, and Iain D Couzin. Emergent sensing of complex environments by mobile animal groups. Science, 339(6119):574-576, 2013.

[4] Roger Bivand, Edzer Pebesma, and Virgillo Gomez-Rubior. Appied Spatial Data Analysis with R. Springer, 2008.

[5] Vincent Brach. Anelosimus studiosus (araneae: Theridiidae) and the evolution of quasisociality in theridiid spiders. Evolution, 31(1):154161, 1977.

[6] Colin W Clark and Marc Mangel. The evolutionary advantages of group foraging. Theoretical Population Biology, 30(1):45-75, 1986.

[7] Noam Cvikel, Katya Egert Berg, Eran Levin, Edward Hurme, Ivailo Borissov, Arjan Boonman, Eran Amichai, and Yossi Yovel. Bats aggregate to improve prey search but might be impaired when their density becomes too high. Current Biology, 25(2):206-211, 2015.

[8] Rainer Foelix. Biology of spiders. Oxford University Press, 2010.

[9] Frank Heppner and Ulf Grenander. Ubiquity of Chaos, chapter A Stochastic Nonlinear Model for Coordinated Bird Flocks, pages 233238. AAAS Publications, Washington, DC, 1990.

[10] Jennifer UM Jarvis, M Justin O'Riain, Nigel C Bennett, and Paul W Sherman. Mammalian eusociality: a family affair. Trends in Ecology $\mathcal{E}$ Evolution, 9(2):47-51, 1994. 
[11] Thomas Jones, Susan Riechert, Sarah Dalrymple, and Patricia Parker. Fostering model explains variation in levels of sociality in a spider system. Animal Behavior, 73(1):195-204, 2007.

[12] Thomas C Jones and Patricia G Parker. Delayed juvenile dispersal benefits both mother and offspring in the cooperative spider anelosimus studiosus (araneae: Theridiidae). Behavioral Ecology, 13(1):142-148, 2002 .

[13] Michele Joyner, Chelsea Ross, and Edith Seier. Distance to the border in spatial point patterns. Spatial Statistics, 6:24-40, 2013.

[14] Ernst J Kullmann. Evolution of social behavior in spiders (araneae; eresidae and theridiidae). American Zoologist, 12(3):419-426, 1972.

[15] Daniel R MacNulty, Aimee Tallian, Daniel R Stahler, and Douglas W Smith. Influence of group size on the success of wolves hunting bison. 2014.

[16] Inc Minitab. MINITAB. Minitab, 2013.

[17] Craig Packer and Lore Ruttan. The evolution of cooperative hunting. American Naturalist, pages 159-198, 1988.

[18] Jonathan N Pruitt and Maud CO Ferrari. Intraspecific trait variants determine the nature of interspecific interactions in a habitat-forming species. Ecology, 92(10):1902-1908, 2011.

[19] Jonathan N Pruitt and Charles J Goodnight. Site-specific group selection drives locally adapted group compositions. Nature, 514(7522):359$362,2014$.

[20] Jonathan N Pruitt and Susan E Riechert. How within-group behavioural variation and task efficiency enhance fitness in a social group. Proceedings of the Royal Society of London B: Biological Sciences, page rspb20101700, 2010.

[21] Jonathan N Pruitt, Susan E Riechert, and Thomas C Jones. Behavioural syndromes and their fitness consequences in a socially polymorphic spider, anelosimus studiosus. Animal Behaviour, 76(3):871-879, 2008. 
[22] Alex J. Quijano, Michele L. Joyner, Edith Seier, Nathaniel Hancock, Michael Largent, and Thomas C. Jones. An aggregate stochastic model incorporating individual dynamics for predation movements of Anelosimus Studiosus. Mathematical Biosciences and Engineering, 12:76-109, 2015.

[23] Susan E Riechert, Rosemarie Roeloffs, and Arthur C Echternacht. The ecology of the cooperative spider agelena consociata in equatorial africa (araneae, agelenidae). Journal of Arachnology, pages 175-191, 1986.

[24] Ankit Rohatgi. Webplotdigitizer. URL http://arohatgi. info/WebPlotDigitizer/app, 2011.

[25] Chelsea Ross. Ontogeny and diel rhythm in spacing within a subsocial web of Anelosimus Studiosus (araneael therididdae). Honor's Thesis, East Tennessee State University, May 2013.

[26] David Scheel and Craig Packer. Group hunting behaviour of lions: a search for cooperation. Animal Behaviour, 41(4):697-709, 1991.

[27] Orr Spiegel, Wayne M Getz, and Ran Nathan. Factors influencing foraging search efficiency: why do scarce lappet-faced vultures outperform ubiquitous white-backed vultures? The American Naturalist, 181(5):E102-E115, 2013.

[28] Colin J Torney, Andrew Berdahl, and Iain D Couzin. Signalling and the evolution of cooperative foraging in dynamic environments. 2011.

[29] Thomas J Valone. Group foraging, public information, and patch estimation. Oikos, pages 357-363, 1989.

[30] Eric C Yip, Kimberly S Powers, and Leticia Avilés. Cooperative capture of large prey solves scaling challenge faced by spider societies. Proceedings of the National Academy of Sciences, 105(33):11818-11822, 2008. 